TRANSACTIONS OF THE

AMERICAN MATHEMATICAL SOCIETY

Volume 365, Number 6, June 2013, Pages 2895-2930

S 0002-9947(2012)05711-4

Article electronically published on December 13, 2012

\title{
THE MIXED PROBLEM IN LIPSCHITZ DOMAINS WITH GENERAL DECOMPOSITIONS OF THE BOUNDARY
}

\author{
J. L. TAYLOR, K. A. OTT, AND R. M. BROWN
}

\begin{abstract}
This paper continues the study of the mixed problem for the Laplacian. We consider a bounded Lipschitz domain $\Omega \subset \mathbf{R}^{n}, n \geq 2$, with boundary that is decomposed as $\partial \Omega=D \cup N$, with $D$ and $N$ disjoint. We let $\Lambda$ denote the boundary of $D$ (relative to $\partial \Omega$ ) and impose conditions on the dimension and shape of $\Lambda$ and the sets $N$ and $D$. Under these geometric criteria, we show that there exists $p_{0}>1$ depending on the domain $\Omega$ such that for $p$ in the interval $\left(1, p_{0}\right)$, the mixed problem with Neumann data in the space $L^{p}(N)$ and Dirichlet data in the Sobolev space $W^{1, p}(D)$ has a unique solution with the non-tangential maximal function of the gradient of the solution in $L^{p}(\partial \Omega)$. We also obtain results for $p=1$ when the Dirichlet and Neumann data come from Hardy spaces, and a result when the boundary data comes from weighted Sobolev spaces.
\end{abstract}

\section{INTRODUCTION}

In this paper we consider the mixed problem, or Zaremba's problem, for the Laplacian. Let $\Omega$ be a bounded Lipschitz domain in $\mathbf{R}^{n}$ and write $\partial \Omega=D \cup N$, where $D$ is an open set of the boundary and $N=\partial \Omega \backslash D$. We define the $L^{p}$-mixed problem as the following boundary value problem:

$$
\begin{cases}\Delta u=0, & \text { in } \Omega, \\ u=f_{D}, & \text { on } D, \\ \frac{\partial u}{\partial \nu}=f_{N}, & \text { on } N, \\ (\nabla u)^{*} \in L^{p}(\partial \Omega) . & \end{cases}
$$

Here, $(\nabla u)^{*}$ stands for the non-tangential maximal function of $\nabla u$. The normal derivative $\partial u / \partial \nu$ is defined as $\nabla u \cdot \nu$, where $\nu$ is the outward unit normal vector defined a.e. on $\partial \Omega$. Throughout the paper, all boundary values of $u$ and $\partial u / \partial \nu$ are defined as non-tangential limits. See Section 2 for precise definitions.

The study of the mixed problem is a natural continuation of the program of study of boundary value problems in Lipschitz domains which began over thirty years ago. Dahlberg [7] treated the Dirichlet problem for the Laplacian in Lipschitz domains, while Jerison and Kenig [15] treated the Neumann problem with boundary data in $L^{2}$ and the regularity problem with Dirichlet data having one derivative in $L^{2}$. Verchota 34 studied the regularity problem with Dirichlet data with one derivative in $L^{p}$, and Dahlberg and Kenig studied the Neumann problem with $L^{p}$ data 9 . The mixed boundary value problem in Lipschitz domains appears as an open problem

Received by the editors May 10, 2011.

2010 Mathematics Subject Classification. Primary 35J25, 35J05.

The second author's research was supported in part by the National Science Foundation.

The third author's research was supported in part by a grant from the Simons Foundation. 
in Kenig's CBMS lecture notes [16, Problem 3.2.15]. There is a large literature on boundary value problems in polyhedral domains, and we do not attempt to summarize this work here. See the work of Băcuţă et al. [1] for recent results for the mixed problem in polyhedral domains and additional references.

Under mild restrictions on the boundary data we can use energy estimates to show that there exists a solution of the mixed problem with $\nabla u$ in $L^{2}$ of the domain. Our goal in this paper is to obtain more regularity of the solution and, in particular, to show that $\nabla u$ lies in $L^{p}(\partial \Omega)$. Brown [2] showed that the solution satisfies $\nabla u \in L^{2}(\partial \Omega)$ when the data $f_{N}$ is in $L^{2}(N)$ and $f_{D}$ is in the Sobolev space $W^{1,2}(D)$ for a certain class of Lipschitz domains. Roughly speaking, his results hold when the Dirichlet and Neumann portions of the boundary meet at an angle strictly less than $\pi$. In this same class of domains, Sykes and Brown 31 obtain $L^{p}$ results for $1<p<2$ and I. Mitrea and M. Mitrea 24] establish well-posedness in an essentially optimal range of function spaces. Lanzani, Capogna and Brown 20] establish $L^{p}$ results in two-dimensional graph domains when the data comes from weighted $L^{2}$-spaces and the Lipschitz constant is less than one. The aforementioned results rely on a variant of the Rellich identity. The Rellich identity cannot be used in the same way in general Lipschitz domains because it produces estimates in $L^{2}$, and even in smooth domains simple examples show that we cannot expect to have solutions with gradient in $L^{2}(\partial \Omega)$.

Ott and Brown [26] establish conditions on $\Omega, N$, and $D$ which ensure uniqueness of solutions of the $L^{p}$-mixed problem, and they also establish conditions on $\Omega, N$, $D$ and $f_{N}$ and $f_{D}$ which ensure that solutions to the $L^{p}$-mixed problem exist. All of this work is done under an additional geometric assumption on the boundary of $D$. More specifically, the authors address solvability of the mixed problem for the Laplacian in bounded Lipschitz domains under the assumption that the boundary between $D$ and $N$ (relative to $\partial \Omega$ ) is locally given by a Lipschitz graph. Under these conditions on $\Omega, N$, and $D$, they prove that there exists $p_{0}>1$ depending on the Lipschitz constant of the domain and on the dimension $n$, such that for $p$ in the interval $1<p<p_{0}$, the $L^{p}$-mixed problem with Neumann data in $L^{p}(N)$ and Dirichlet data in the Sobolev space $W^{1, p}(D)$ has a solution and this solution is unique in the class of functions satisfying $(\nabla u)^{*} \in L^{p}(\partial \Omega)$. In the case $p=$ 1 , they prove results for the mixed problem with data from Hardy spaces. The novelty of this paper is to address existence and uniqueness of solutions of the $L^{p_{-}}$ mixed problem under more general conditions on the decomposition of the boundary into sets $N$ and $D$. Our proof relies on a technique of Shen [28] to use reverse Hölder inequalities to establish existence of solutions to the $L^{p}$-mixed problem. This technique allows for an immediate extension to the mixed boundary value problem with data from weighted spaces. We carry out a study of the mixed problem in weighted spaces in Section 7. As one step of this argument, we need to consider the regularity problem with boundary data in weighted $L^{p}$-spaces.

The boundary between $D$ and $N$ is an important feature in the study of the mixed problem. Assume that $D$ is a relatively open subset of $\partial \Omega$ and let $\Lambda$ denote the boundary of $D$ (relative to $\partial \Omega$ ). Before stating our assumptions on $\Omega, N$, and $D$, we introduce the following notation. We will use $\delta(y)=\operatorname{dist}(y, \Lambda)$ to denote the distance from a point $y$ to $\Lambda$. Let $B_{r}(x)=\{y:|y-x|<r\}$ denote the standard ball in $\mathbf{R}^{n}$ and let $\Psi_{r}(x)=B_{r}(x) \cap \Omega$. For $x \in \bar{\Omega}$, let $\Delta_{r}(x)=B_{r}(x) \cap \partial \Omega$ denote a surface ball. We note that the term surface ball is not ideal since the "center" $x$ may 
not lie on the boundary. In addition, we will need to be careful in places because $\Delta_{r}(x)$ may not be a connected set. See Section 2 for other relevant definitions.

Our assumptions on $\Omega$ and $D$ are stated here. We will obtain results only when the parameter $\epsilon$ in (1.3) is small. See Section 2 for a definition of the constant $r_{0}$.

$$
\Omega \subset \mathbf{R}^{n}, n \geq 2 \text {, is a bounded Lipschitz domain of constant } M .
$$

The set $\Lambda$ is an Ahlfors $(n-2+\epsilon)$-regular set: There exists $M>0$ such that

$$
\mathcal{H}^{n-2+\epsilon}\left(\Delta_{r}(x) \cap \Lambda\right) \leq M r^{n-2+\epsilon}, \quad \text { for all } x \in \Lambda, 0<r<r_{0},
$$

with $\epsilon \geq 0$. The notation $\mathcal{H}^{n-2+\epsilon}(E)$ denotes the $(n-2+\epsilon)$-dimensional Hausdorff measure of a set $E$. Our third main assumption is that the set $D$ satisfies the corkscrew condition relative to $\partial \Omega$ : There exists $M>0$ such that

$$
\begin{aligned}
& \text { for all } x \in \Lambda, 0<r<r_{0} \text {, there exists } \tilde{x} \in D \text { such that }|x-\tilde{x}|<r \\
& \text { and } \delta(\tilde{x})>M^{-1} r \text {. }
\end{aligned}
$$

Several previously studied cases of the mixed problem fall under the conditions of assumptions (1.2), (1.3), and (1.4). Venouziou and Verchota 32 establish a solution to the $L^{p}$-mixed problem (1.1) in polyhedral domains in $\mathbf{R}^{3}$. In one particular case, they are able to solve the mixed boundary value problem in the pyramid in $\mathbf{R}^{3}$ when Dirichlet and Neumann data are assigned to alternating faces. This example is not covered by the earlier work of Ott and Brown [26] because, at the apex of the pyramid, the boundary between $D$ and $N$ is not locally given by a Lipschitz graph. The pyramid example is covered by the results in this paper. Another example that is covered by this work, but not the earlier work of Ott and Brown [26], is the case where the boundary of $D$ is a Koch snowflake of dimension slightly greater than $n-2$.

We now state the main theorem of the paper. The definitions are given in Section 2 Since we do not assume that the Dirichlet set $D \subset \partial \Omega$ is an extension domain for Sobolev spaces, note that we must assume that the Sobolev space $W^{1, p}(D)$ is defined by restricting elements in $W^{1, p}(\partial \Omega)$ to $D$. See Section 2 for a discussion of the constants in the estimates of this theorem.

Theorem 1.1. Let $\Omega$ and $D$ satisfy conditions (1.2), (1.3), and (1.4). There exists an exponent $q_{0}>2$, which depends on $M$ and $n$, such that if $\Lambda$ satisfies (1.3), with $0 \leq \epsilon<\left(q_{0}-2\right) /\left(q_{0}-1\right)$, the following statements are true.

a) If $p \geq 1$, the $L^{p}$-mixed problem has at most one solution.

b) If $f_{N}$ lies in $H^{1}(N)$ and $f_{D}$ lies in $H^{1,1}(D)$, the $L^{1}$-mixed problem has a solution $u$ which satisfies the estimate

$$
\left\|(\nabla u)^{*}\right\|_{L^{1}(\partial \Omega)} \leq C\left(\left\|f_{N}\right\|_{H^{1}(N)}+\left\|f_{D}\right\|_{H^{1,1}(D)}\right) .
$$

c) If $p_{0}=q_{0}((1-\epsilon) /(2-\epsilon))>1$, then for $p$ in the interval $\left(1, p_{0}\right)$ the following holds: If $f_{N}$ lies in $L^{p}(N)$ and $f_{D}$ lies in $W^{1, p}(D)$, then the $L^{p}$-mixed problem has a solution $u$ which satisfies

$$
\left\|(\nabla u)^{*}\right\|_{L^{p}(\partial \Omega)} \leq C\left(\left\|f_{N}\right\|_{L^{p}(N)}+\left\|f_{D}\right\|_{W^{1, p}(D)}\right) .
$$

The proof of the main theorem will proceed as follows. We begin by recognizing that it suffices to prove Theorem 1.1 in the case where the Dirichlet data is zero. This is because non-tangential maximal function estimates for the gradient of the solution to the Dirichlet problem are known. When the Dirichlet data comes from a Sobolev space, these estimates were treated for $p=2$ by Jerison and Kenig [15] 
and for $1<p<2$ by Verchota [33, 34. The case of the Dirichlet problem with data from a Hardy space was treated by Dahlberg and Kenig 9 and by D. Mitrea in two dimensions [23, Theorem 3.6].

The first main result presented in this paper is the existence of solutions of the mixed problem when the Neumann data is an atom for a Hardy space. The proof of this result is contained in Section 4. The key ingredient of the proof is an estimate of the Green function for the mixed problem, which is proved in Section 3. In Section 5 we prove uniqueness of solutions to the $L^{p}$-mixed problem, $p \geq 1$. Section 6 contains the proof of the $L^{p}$ result, and Section 7 contains the proof of the weighted result.

\section{Preliminaries}

Throughout the paper, we will work under the assumption (1.2) that $\Omega$ is a bounded Lipschitz domain. A bounded, connected open set $\Omega$ is called a Lipschitz domain with Lipschitz constant $M$ if locally $\Omega$ is a domain which lies above the graph of a Lipschitz function. More precisely, for $M>0, x \in \partial \Omega$, and $r>0$, define a coordinate cylinder $Z_{r}(x)$ to be $Z_{r}(x)=\left\{y:\left|y^{\prime}-x^{\prime}\right|<r,\left|y_{n}-x_{n}\right|<(1+M) r\right\}$. Use coordinates $\left(x^{\prime}, x_{n}\right) \in \mathbf{R}^{n-1} \times \mathbf{R}$ and assume that this coordinate system is a translation and rotation of the standard coordinates. Then $\Omega$ is a Lipschitz domain if for each $x \in \partial \Omega$ there exists a coordinate cylinder and a Lipschitz function $\phi: \mathbf{R}^{n-1} \rightarrow \mathbf{R}$ with Lipschitz constant $M$ such that

$$
\begin{aligned}
\Omega \cap Z_{r}(x) & =\left\{\left(y^{\prime}, y_{n}\right): y_{n}>\phi\left(y^{\prime}\right)\right\} \cap Z_{r}(x), \\
\partial \Omega \cap Z_{r}(x) & =\left\{\left(y^{\prime}, y_{n}\right): y_{n}=\phi\left(y^{\prime}\right)\right\} \cap Z_{r}(x) .
\end{aligned}
$$

Fix a covering of the boundary by coordinate cylinders $\left\{Z_{r_{i}}\left(x_{i}\right)\right\}_{i=1}^{L}$ such that each $Z_{100 r_{i} \sqrt{1+M^{2}}}\left(x_{i}\right)$ is also a coordinate cylinder. Let $r_{0}=\min \left\{r_{i}: i=1, \ldots, L\right\}$.

For a Lipschitz domain $\Omega$ we define a decomposition of the boundary for the mixed problem, $\partial \Omega=D \cup N$, as follows. Assume that $D$ is a relatively open subset of $\partial \Omega$, $N=\partial \Omega \backslash D$, and let $\Lambda$ be the boundary of $D$ (relative to $\partial \Omega$ ). The assumptions on the decomposition of the boundary for the mixed problem are given in (1.3) and (1.4). Recall that $\delta(y)=\operatorname{dist}(y, \Lambda)$ denotes the distance from a point $y$ to the boundary of $D$.

Many of our estimates will be of a local, scale invariant nature and will hold for $r$ less than a multiple of $r_{0}$, and with a constant that depends only on the constant $M$ in assumptions (1.2)-(1.4), $\epsilon$ in (1.3), the dimension $n$, and any $L^{p}$-indices that appear in the estimate. We say that an estimate depends on the global character of the domain if it depends on the above and also on the collection of coordinate cylinders which cover $\partial \Omega$ and the constant in the coercivity condition (3.2). The notation $A \approx B$ will mean that $c^{-1} B \leq A \leq c B$ for some constant $c$ depending only on $M$ and $n$.

We now prove several consequences of conditions (1.3) and (1.4) that we will appeal to later in the paper.

Lemma 2.1. Let $\Omega$ satisfy (1.2) and let $r$ be such that $0<r<r_{0}$. If $x \in \partial \Omega$ satisfies $\delta(x) \geq r \sqrt{1+M^{2}}$, then $\Delta_{r}(x) \subset N$ or $\Delta_{r}(x) \subset D$.

Proof. Let $x \in \partial \Omega$ and fix $y \in \Delta_{r}(x)$. Since $0<r<r_{0}$, we may find a coordinate cylinder $Z$ which contains $\Delta_{r}(x)$. Let $\phi$ be the function whose graph gives $\partial \Omega$ near $Z$. Since $y \in \Delta_{r}(x)$, we have that $\left|y^{\prime}-x^{\prime}\right|<r$. Now define a function 
$x^{\prime}:[0,1] \rightarrow \mathbf{R}^{n-1}, x^{\prime}(t)=(1-t) x^{\prime}+t y^{\prime}$. Then $\gamma(t)=\left(x^{\prime}(t), \phi\left(x^{\prime}(t)\right)\right)$ gives a path contained in $\partial \Omega$ that connects $x$ and $y$ and has length less than $r \sqrt{1+M^{2}}$. Since $\delta(x) \geq r \sqrt{1+M^{2}}$ and $\delta$ is Lipschitz with constant one, we have that $\delta(\gamma(t))>0$ for $0 \leq t \leq 1$. Since $\gamma(t)$ does not pass through $\Lambda$, we have that both $x$ and $y$ must lie in either $D$ or $N$. The point $y$ was arbitrarily chosen in $\Delta_{r}(x)$; therefore $\Delta_{r}(x) \subset D$ or $\Delta_{r}(x) \subset N$.

The following lemma is adapted from a result found in Lehrbäck [21, pp. 254255]. Here and throughout the paper, we use $\sigma$ to denote surface measure.

Lemma 2.2. Let $\Omega$ and $D$ satisfy (1.2) and (1.3) and let $r$ satisfy $0<r<r_{0}$. Then for each $x \in \Lambda$ and $0<t<2 r$,

$$
\sigma\left(\Delta_{r}(x) \cap\{y: \delta(y)<t\}\right) \leq C t^{1-\epsilon} r^{n-2+\epsilon} .
$$

Proof. Fix $x \in \Lambda, 0<r<r_{0}$ and $t$ with $0<t<2 r$. By a standard covering lemma, there exists a finite, disjoint collection of surface balls $\left\{\Delta_{t}\left(y_{i}\right)\right\}$ with $y_{i} \in \Delta_{r}(x) \cap \Lambda$, $i=1,2, \ldots, m$, such that $\left(\Delta_{r}(x) \cap \Lambda\right) \subset \bigcup_{i=1}^{m} \Delta_{3 t}\left(y_{i}\right)$. Using that the collection of balls $\left\{\Delta_{t}\left(y_{i}\right)\right\}_{i=1}^{m}$ is disjoint and the $(n-2+\epsilon)$-regularity of $\Lambda$ given in (1.3), we have

$$
\begin{aligned}
t^{n-2+\epsilon} m & \leq C \sum_{i=1}^{m} \mathcal{H}^{n-2+\epsilon}\left(\Delta_{t}\left(y_{i}\right) \cap \Lambda\right) \\
& \leq C \mathcal{H}^{n-2+\epsilon}\left(\Delta_{3 r}(x) \cap \Lambda\right) \\
& \leq C r^{n-2+\epsilon}
\end{aligned}
$$

This calculation yields the estimate $m \leq m_{t}=C(r / t)^{n-2+\epsilon}$ for $t<2 r$, where $C$ depends on $M$ and the dimension $n$.

Now,

$$
\begin{aligned}
\sigma\left(\Delta_{r}(x) \cap\{y: \delta(y)<t\}\right) & \leq C \sum_{i=1}^{m_{t}} \sigma\left(\Delta_{4 t}\left(y_{i}\right)\right) \\
& \leq C t^{n-1} m_{t} \\
& \leq C t^{n-1}\left(\frac{r}{t}\right)^{n-2+\epsilon}=C t^{1-\epsilon} r^{n-2+\epsilon},
\end{aligned}
$$

which proves the lemma.

Lemma 2.3. Let $\Omega$ and $D$ satisfy (1.2) and (1.4). If $x \in D$ and $0<r<r_{0}$, then there exists a constant $c$ and $x_{D} \in D$ such that $\left|x-x_{D}\right| \leq r$ and $\Delta_{c r}\left(x_{D}\right) \subset D$. Furthermore,

$$
\sigma\left(\Delta_{r}(x) \cap D\right) \geq c r^{n-1} .
$$

Proof. Let $x \in D$ and $0<r<r_{0}$. We will break up the proof into two cases. First suppose that $r / 2<\delta(x)$, and let $x_{D}=x$. Let $Z_{r}\left(x_{D}\right)$ be the coordinate cylinder centered at $x_{D}$ with radius $r$. Using Lemma 2.1 we see that $\delta\left(x_{D}\right)>r / 2$ implies that $\Delta_{c_{1} r}(x) \subset D$ for $c_{1}=1 /\left(2 \sqrt{1+M^{2}}\right)$.

Now consider the case where $\delta(x) \leq r / 2$. According to (1.4) and Lemma 2.1. given $\hat{x} \in \Lambda$, there exists $\bar{x} \in D$ such that $|\hat{x}-\bar{x}| \leq r / 2$ and $\Delta_{c_{2} r}(\bar{x}) \subset D$ with $c_{2}=1 /\left(2 M \sqrt{1+M^{2}}\right)$. Recall that $\delta(x) \leq r / 2$, and choose $\hat{x}$ on $\Lambda$ so that 
$|x-\hat{x}|=\delta(x)$. By the remark above, there exists $\bar{x}$ with $|\hat{x}-\bar{x}| \leq r / 2$ and $\Delta_{c_{1} r}(\bar{x}) \subset D$. Let $x_{D}=\bar{x}$. Then

$$
\begin{aligned}
\left|x-x_{D}\right| & \leq|x-\hat{x}|+\left|\hat{x}-x_{D}\right| \\
& \leq r .
\end{aligned}
$$

Thus, we obtain $\Delta_{c r}(x) \subset D$ if $c \leq \min \left(c_{1}, c_{2}\right)$. Conclusion (2.2) is an immediate consequence.

The next two lemmas establish integrability of the distance function $\delta$.

Lemma 2.4. Let $\Omega$ and $D$ satisfy (1.2), (1.3), and (1.4). If $x \in \partial \Omega$ and $r$ satisfies $0<r<r_{0}$, then for $-1+\epsilon<s<\infty$,

$$
\int_{\Delta_{r}(x)} \delta(y)^{s} d \sigma \approx r^{n-1} \max (r, \delta(x))^{s} .
$$

Proof. Fix $x \in \partial \Omega, r$ in the interval $\left(0, r_{0}\right)$ and consider several cases: a) $s \geq 0$ and $\delta(x)<r / 4, \mathrm{~b}) s \geq 0$ and $\delta(x) \geq r / 4$, c) $s<0$ and $\delta(x)<4 r$, and d) $s<0$ and $\delta(x) \geq 4 r$.

Case a) Assume that $s \geq 0, \delta(x)<r / 4$. Since $\delta(x)<r / 4$ and $\delta$ is Lipschitz with constant one, we have $\delta(y) \leq 5 r / 4$ for all $y \in \Delta_{r}(x)$. As $\partial \Omega$ is the boundary of a Lipschitz domain, we have $\sigma\left(\Delta_{r}(x)\right) \approx r^{n-1}$. Thus when $s \geq 0$, the upper bound $\int_{\Delta_{r}(x)} \delta(y)^{s} d \sigma \leq C r^{n-1+s}$ follows easily.

To obtain a lower bound, we begin by finding $\hat{x} \in \Lambda$ such that $|x-\hat{x}|=\delta(x)<$ $r / 4$. Then the corkscrew condition for $D$ (1.4) gives a point $\tilde{x}$ with $|x-\tilde{x}|<$ $r / 4$ and $\delta(\tilde{x})>r /(4 M)$. If $y \in \Delta_{r /(8 M)}(\tilde{x})$ we have that $\delta(y) \geq r /(8 M)$, and $\Delta_{r /(8 M)}(\tilde{x}) \subset \Delta_{r}(x)$ since $|x-\tilde{x}|<r / 2$. Thus the lower bound $\int_{\Delta_{r}(x)} \delta(y)^{s} d \sigma \geq$ $\int_{\Delta_{r /(8 M)}(\tilde{x})} \delta(y)^{s} d \sigma \geq C r^{n-1+s}$ follows.

Case b) Assume that $s \geq 0, \delta(x) \geq r / 4$. Observe that if $y \in \Delta_{r}(x)$, then $\delta(y) \leq \delta(x)+r \leq 5 \delta(x)$ and the upper bound $\int_{\Delta_{r}(x)} \delta(y)^{s} d \sigma \leq r^{n-1} \delta(x)^{s}$ follows.

To obtain the lower bound, we use that if $\delta(x) \geq r / 4$ and $y \in \Delta_{r / 8}(x)$, then $\delta(x) \leq 2 \delta(y)$, and hence we have $\int_{\Delta_{r}(x)} \delta(y)^{s} d \sigma \geq \int_{\Delta_{r / 8}(x)} \delta(y)^{s} d \sigma \geq c r^{n-1} \delta(x)^{s}$.

Case c) Assume that $-1+\epsilon<s<0, \delta(x)<4 r$. We divide the surface ball $\Delta_{r}(x)$ using level sets of the distance function and then use Lemma 2.2 to obtain

$$
\begin{aligned}
\int_{\Delta_{r}(x)} \delta(y)^{s} d \sigma & =\sum_{k=0}^{\infty} \int_{\Delta_{r}(x) \cap\left\{y: 2^{-k-1} r<\delta(y) \leq 2^{-k} r\right\}} \delta(y)^{s} d \sigma \\
& \leq C \sum_{k=0}^{\infty} r^{n-2+\epsilon}\left(2^{-k} r\right)^{s+1-\epsilon} \\
& \leq C r^{n-1+s} .
\end{aligned}
$$

In the last inequality above we use the assumption that $s>-1+\epsilon$ to sum the geometric series.

To obtain the lower bound, we observe that if $\delta(x) \leq 4 r$, then for $y \in \Delta_{r}(x)$, $\delta(y) \leq \delta(x)+r<5 r$. Since $s<0$, it follows that $\delta(y)^{s} \geq(5 r)^{s}$, and the lower bound $\int_{\Delta_{r}(x)} \delta(y)^{s} d \sigma \geq C r^{n-1+s}$ follows easily.

Case d) Assume that $-1+\epsilon<s<0, \delta(x) \geq 4 r$. If $y \in \Delta_{r}(x)$, then we have $3 \delta(x) / 4 \leq \delta(y) \leq 5 \delta(x) / 4$. Thus, we obtain $\int_{\Delta_{r}(x)} \delta(y)^{s} d \sigma \approx r^{n-1} \delta(x)^{s}$.

The result of the lemma follows easily from the four cases above. 
The integrability of the function $\delta$ over interior balls is a straightforward adaptation of the previous result.

Lemma 2.5. Let $\Omega$ and $D$ satisfy (1.2), (1.3), and (1.4), and let $r$ satisfy $0<r<$ $r_{0}$. Then for $s \in(-2+\epsilon, \infty)$,

$$
\int_{\Psi_{r}(x)} \delta(y)^{s} d y \approx r^{n} \max (r, \delta(x))^{s} .
$$

Throughout this work, the main tool for estimating solutions will be the nontangential maximal function. Fix $\alpha>0$, and for $x \in \partial \Omega$, the non-tangential approach region is defined by

$$
\Gamma(x)=\{y \in \Omega:|x-y| \leq(1+\alpha) \operatorname{dist}(y, \partial \Omega)\} .
$$

Given a function $u$ defined on $\Omega$, the non-tangential maximal function is defined as

$$
u^{*}(x)=\sup _{y \in \Gamma(x)}|u(y)|, \quad x \in \partial \Omega .
$$

We will also utilize a truncated non-tangential approach region,

$$
\Gamma_{r}(x)=\Gamma(x) \cap B_{r}(x),
$$

and, respectively, a truncated non-tangential maximal function,

$$
u_{r}^{*}(x)=\sup _{y \in \Gamma_{r}(x)}|u(y)|, \quad x \in \partial \Omega .
$$

It is well known that for different values of $\alpha$, the non-tangential maximal functions have comparable $L^{p}$-norms. Thus, we suppress the value of $\alpha$ in our notation.

The restrictions of $u$ and $\nabla u$ to the boundary in (1.1) are understood as nontangential limits. Precisely, for a function $v$ defined on $\Omega$ and $x \in \partial \Omega$,

$$
v(x)=\lim _{\Gamma(x) \ni y \rightarrow x} v(y),
$$

provided that the limit exists. It is well known that for a Lipschitz domain $\Omega$ and $v$ a harmonic function in $\Omega$, the non-tangential limits exist at almost every point where the non-tangential maximal function is finite.

We now recall the definitions of atoms and atomic Hardy spaces. A function $a$ is an atom for $\partial \Omega$ if $\operatorname{supp} a \subset \Delta_{r}(x)$ for some $x \in \partial \Omega,\|a\|_{L^{\infty}(\partial \Omega)} \leq 1 / \sigma\left(\Delta_{r}(x)\right)$ and $\int_{\partial \Omega} a d \sigma=0$. In our treatment of the mixed problem, we will consider atoms for the subset $N \subset \partial \Omega$. We say that $\tilde{a}$ is an atom for $N$ if $\tilde{a}$ is the restriction to $N$ of a function $a$ which is an atom for $\partial \Omega$. The Hardy space $H^{1}(N)$, where $N \subset \partial \Omega$, is the collection of functions $f$ which can be represented as $\sum_{j} \lambda_{j} a_{j}$, where each $a_{j}$ is an atom for $N$ and the coefficients $\lambda_{j}$ satisfy $\sum_{j}\left|\lambda_{j}\right|<\infty$. In the case where $N=\partial \Omega$ this definition gives the standard definition of the Hardy space $H^{1}(\partial \Omega)$. The Hardy-Sobolev space $H^{1,1}(\partial \Omega)$ is defined as the set of functions with one derivative in $H^{1}(\partial \Omega)$. More precisely, we say that a function $A$ is an atom for $H^{1,1}(\partial \Omega)$ if $A$ is supported in a surface ball $\Delta_{r}(x)$ for some $x \in \partial \Omega$ and $\left\|\nabla_{t} A\right\|_{L^{\infty}(\partial \Omega)} \leq 1 / \sigma\left(\Delta_{r}(x)\right)$. If $v$ is a smooth function defined in a neighborhood of $\partial \Omega$, then the tangential gradient of $v$ is defined as $\nabla_{t} v=\nabla v-(\nabla v \cdot \nu) \nu$, where $\nu$ is the outward unit normal vector. Then $\tilde{A}$ is an atom for $H^{1,1}(D)$ if $\tilde{A}$ is the restriction to $D$ of an atom $A$ in $H^{1,1}(\partial \Omega)$. The space $H^{1,1}(D)$ is the collection of all functions which can be represented as $\sum_{j} \lambda_{j} A_{j}$, where each $A_{j}$ is an element of $H^{1,1}(D)$ and $\sum_{j}\left|\lambda_{j}\right|<\infty$. 
Finally, we define the Sobolev space $W^{1, p}(\partial \Omega)$ to be the collection of functions in $L^{p}(\partial \Omega)$ whose tangential gradient also lies in $L^{p}(\partial \Omega)$.

\section{Green function estimates And Reverse Hölder inequalities}

An important step in the proof of the main theorem is to show decay of the solution to the mixed problem with Neumann data an $H^{1}(N)$ atom as we move away from the support of the atom. This decay is encoded in estimates for the Green function for the mixed problem, which are proved in this section. The argument that ensues only requires that $\Omega$ be a Lipschitz domain and that $D$ satisfies (2.2).

When working near the boundary, we will want to assume that part of the boundary is flat. This can always be arranged in a Lipschitz domain by flattening the boundary with a change of coordinates. Since flattening the boundary will change the coefficients, we need to consider operators $L$ with bounded and measurable coefficients. Assume that $L=\operatorname{div} A \nabla$, and assume that the coefficient matrix $A$ is real, bounded, and measurable, satisfies $A^{t}=A$, and satisfies the ellipticity condition that there exists a $\lambda>0$ such that

$$
\lambda|\xi|^{2} \leq A \xi \cdot \xi \leq \lambda^{-1}|\xi|^{2} \quad \xi \in \mathbf{R}^{2} .
$$

The optimal $\lambda$ for which the above condition holds is called the ellipticity constant for $L$.

We now define a weak formulation of the mixed problem for solutions of divergence form operators whose gradients lie in $L^{2}(\Omega)$. Our goal is to prove that under appropriate assumptions on the data, the weak solution will have a gradient in $L^{p}(\partial \Omega)$ for $1<p<p_{0}$, for some $p_{0}>1$. For $k=1,2, \ldots, W^{k, p}(\Omega)$ denotes the Sobolev space of functions having $k$ derivatives in $L^{p}(\Omega)$. For $D$ an open subset of the boundary, let $W_{D}^{1,2}(\Omega)$ be the closure in $W^{1,2}(\Omega)$ of functions in $C_{0}^{\infty}\left(\mathbf{R}^{n}\right)$ whose support is disjoint from $\bar{D}$. Let $W_{D}^{1 / 2,2}(\partial \Omega)$ be the restrictions to $\partial \Omega$ of functions in $W_{D}^{1,2}(\Omega)$ and define $W_{D}^{-1 / 2,2}(\partial \Omega)$ to be the dual of $W_{D}^{1 / 2,2}(\partial \Omega)$. We assume that the Dirichlet data is zero and the Neumann data $f_{N}$ lies in the space $W_{D}^{-1 / 2,2}(\partial \Omega)$.

Consider the mixed problem

$$
\begin{cases}\operatorname{div} A \nabla u=0, & \text { in } \Omega, \\ u=0, & \text { on } D, \\ A \nabla u \cdot \nu=f_{N}, & \text { on } N .\end{cases}
$$

We say that $u$ is a weak solution of the problem (3.1) if $u \in W_{D}^{1,2}(\Omega)$ and

$$
\int_{\Omega} A \nabla u \cdot \nabla \phi d y=\left\langle f_{N}, \phi\right\rangle, \quad \phi \in W_{D}^{1,2}(\Omega) .
$$

To establish the existence of weak solutions to the mixed problem, we assume the coercivity condition

$$
\|u\|_{L^{2}(\Omega)} \leq c\|\nabla u\|_{L^{2}(\Omega)}, \quad u \in W_{D}^{1,2}(\Omega) .
$$

Under this assumption, the existence and uniqueness of weak solutions to the boundary value problem (3.1) are a consequence of the Lax-Milgram theorem. In our applications, $\Omega$ will be a connected, bounded Lipschitz domain and $D$ will be an open subset of the boundary. These assumptions are sufficient to ensure that (3.2) holds.

We also need to define a weak solution of the mixed problem on a subset of $\Omega$. Let $\Omega^{\prime}$ be an open subset of $\Omega$. We say that $u$ is a weak solution to $L u=f$ in $\Omega^{\prime}$ 
with zero boundary data for the mixed problem on $\partial \Omega^{\prime} \cap \partial \Omega$ if $u \in W_{\partial \Omega^{\prime} \cap D}^{1,2}\left(\Omega^{\prime}\right)$, and for each test function $\phi$ which lies in $W_{\partial \Omega^{\prime} \cap(D \cup \Omega)}^{1,2}\left(\Omega^{\prime}\right)$, we have

$$
\int_{\Omega^{\prime}} A \nabla u \cdot \nabla \phi d y=-\int_{\Omega^{\prime}} f \phi d y .
$$

Solutions of the mixed problem are bounded. If $u$ is a solution of $L u=0$ in the domain $\Psi_{r}(x)=B_{r}(x) \cap \Omega, x \in \Omega$, and $u$ has zero data for the mixed problem on $\partial \Omega \cap \partial \Psi_{r}(x)$, then there exists a constant $C>0$ such that

$$
|u(x)| \leq C f_{\Psi_{r}(x)}|u(y)| d y .
$$

This may be proved by the Moser iteration method 25, for example.

Finally, we give an estimate on the boundary Hölder continuity of solutions of the mixed problem. For this estimate we consider domains $\Psi_{r}(x)=B_{r}(x)$ with $x \in \Omega$ and $r<\operatorname{dist}(x, \partial \Omega)$, or $\Psi_{r}(x)=B_{r}(x) \cap \Omega$ with $x \in \partial \Omega$ and $r<r_{0}$. In the second case, we assume that $\partial \Omega \cap B_{r}(x)$ lies in a hyperplane. A study of Hölder continuity of solutions of elliptic equations may be found in work of Stampacchia 29]. Stampacchia gives a general framework for studying Hölder continuity of solutions using the method of De Giorgi [10. This framework is applied to the mixed problem in the case where the boundary of $D$ is the bi-Lipschitz image of a hyperplane of co-dimension 2. Our assumptions allow for more general subdivisions of the boundary.

Theorem 3.1. Let $x \in \partial \Omega$ and assume that $0<r<r_{0}$. Let $u$ be a weak solution of the mixed problem in $\Psi_{r}(x)$ with zero data for the mixed problem on $\partial \Psi_{r}(x) \cap \partial \Omega$. Then there exists an exponent $\beta>0$ such that

$$
|u(z)-u(y)| \leq C\left(\frac{|z-y|}{r}\right)^{\beta} \sup _{\Psi_{r}(x)}|u(y)|, \quad z, y \in \Psi_{r / 2}(x) .
$$

The constant $C$ and the exponent $\beta$ depend only on the ellipticity constant $\lambda$ as well as $M$ and $n$.

The proof will follow the method of de Giorgi [10] as given in the monograph of Ladyzhenskaya and Ural'tseva [19]. Fix $\Psi_{R}\left(x_{0}\right)$ as above. We say that a bounded function $u$ lies in the space $\mathcal{B}\left(\Psi_{R}\left(x_{0}\right), \gamma\right)$ if for each $\Psi_{s}(x) \subset \Psi_{R}\left(x_{0}\right), \sigma \in(0,1)$ and $k$ as below we have

$$
\int_{A_{k, s-\sigma s}}|\nabla u|^{2} d y \leq \frac{\gamma}{\sigma^{2} s^{2}} \sup _{A_{k, s}}(u-k)^{2}\left|A_{k, s}\right| .
$$

Here, $A_{k, s}=\left\{y \in \Psi_{s}(x): u(y)>k\right\}$ with $k$ an arbitrary real number if $\partial \Psi_{s}(x) \cap$ $D=\emptyset$ and $k \geq 0$ if $\partial \Psi_{s}(x) \cap D \neq \emptyset$.

Lemma 3.2. Let $x \in \partial \Omega$ and let $r$ satisfy $0<r<r_{0}$. If $u$ is a solution of the elliptic operator $L$ in $\Psi_{r}(x)$ with zero data for the mixed problem on $\partial \Psi_{r}(x) \cap \partial \Omega$, then $u \in \mathcal{B}\left(\Psi_{r}(x), \gamma\right)$ and $\gamma$ depends only on the ellipticity constant for $L$.

Proof. Fix $\Psi_{s}(y)$, which is contained in $\Psi_{r}(x)$ and $\sigma \in(0,1)$. Let $\eta$ be a smooth cutoff function that is supported in $B_{s}(y)$ with $\eta=1$ on $B_{s-\sigma s}(y)$ and satisfies $|\nabla \eta| \leq C_{0} /(\sigma s)$. Let $u^{+}=\max (u, 0)$ denote the positive part of a function $u$. If $k$ 
is as in the definition of the space $\mathcal{B}$, then $(u-k)^{+} \eta^{2}$ may be used as a test function in the weak formulation of $\operatorname{div} A \nabla u=0$, and thus we have

$$
\int_{\Psi_{s}(y)} \eta^{2} A \nabla u \cdot \nabla(u-k)^{+} d y=-2 \int_{\Psi_{s}(y)} \eta(u-k)^{+} A \nabla u \cdot \nabla \eta d y .
$$

Using the symmetry and non-negativity of $A$ and Young's inequality, we obtain

$$
\int_{A_{k, s}} \eta^{2} A \nabla u \cdot \nabla u d y \leq \int_{A_{k, s}} \frac{1}{2} \eta^{2} A \nabla u \cdot \nabla u+2(u-k)^{2} A \nabla \eta \cdot \nabla \eta d y .
$$

Subtracting the first term on the right and using the ellipticity of $A$ gives

$$
\frac{\lambda}{2} \int_{A_{k, s}} \eta^{2}|\nabla u|^{2} d y \leq \frac{2}{\lambda} \int_{A_{k, s}}(u-k)^{2}|\nabla \eta|^{2} d y .
$$

Recalling the estimate $|\nabla \eta| \leq C_{0} /(\sigma s)$ and that $\eta=1$ on $B_{s-\sigma s}(y)$, we conclude that

$$
\int_{A_{k, s-\sigma s}}|\nabla u|^{2} d y \leq \frac{\gamma}{(\sigma s)^{2}}\left|A_{k, s}\right| \sup _{A_{k, s}}(u-k)^{2} .
$$

Thus $u$ is in the space $\mathcal{B}\left(\Psi_{r}(x), \gamma\right)$ with $\gamma=4 C_{0}^{2} / \lambda^{2}$.

Lemma 3.3. Let $x \in \partial \Omega$ and assume that $0<r<r_{0}$. If $u \in \mathcal{B}\left(\Psi_{r}(x), \gamma\right)$ and $k$ is as in the definition of this space, then there exists $\theta_{1}=\theta_{1}(n, \gamma)$ such that if $\left|A_{k, r}\right| \leq \theta_{1} r^{n}$ and $H=\sup _{A_{k, r}}(u-k)>0$, then $\left|A_{k+H / 2, r / 2}\right|=0$ and hence

$$
\sup _{\Psi_{r / 2}(x)}(u-k) \leq H / 2 .
$$

Before giving the proof of Lemma 3.3. we need to give two versions of the SobolevPoincaré inequality.

Lemma 3.4. Let $x \in \Omega$ and let $r$ satisfy $0<r<r_{0}$. If $u \in W^{1,1}\left(\Psi_{r}(x)\right)$ and $\ell>k$, then

$$
(\ell-k)\left|A_{\ell, r}\right|^{1-1 / n} \leq C \frac{\left|\Psi_{r}(x)\right|}{\left|\Psi_{r}(x) \backslash A_{k, r}\right|} \int_{A_{k, r} \backslash A_{\ell, r}}|\nabla u| d y .
$$

If $B_{r / 2}(x) \cap D \neq \emptyset, u \in W^{1,1}\left(\Psi_{r}(x)\right)$, and $k \geq 0$, then

$$
(\ell-k)\left|A_{\ell, r}\right|^{1-1 / n} \leq C \int_{A_{k, r} \backslash A_{\ell, r}}|\nabla u| d y .
$$

Proof. In each case, the estimate follows by applying a Sobolev inequality to the function

$$
v(y)= \begin{cases}(u(y)-k)^{+}, & u(y) \leq \ell, \\ \ell-k, & u(y)>\ell .\end{cases}
$$

The estimate (3.5) uses our assumption that $D$ satisfies (2.2). See Section 3 of [26], for example.

Proof of Lemma 3.3. Fix $\Psi_{r}(x)$ and let $u \in \mathcal{B}\left(\Psi_{r}(x), \gamma\right)$. For $h=0,1,2, \ldots$, set

$$
r_{h}=r / 2+r /\left(2^{h+1}\right) \quad \text { and } \quad k_{h}=k+H / 2-H /\left(2^{h+1}\right),
$$

where $H$ is as in the statement of Lemma 3.3. We will use the notation $\sigma_{h}=$ $\left(r_{h}-r_{h+1}\right) / r_{h}$. Since $u \in \mathcal{B}\left(\Psi_{r}(x), \gamma\right)$, we have

$$
\int_{A_{k_{h}, r_{h+1}}}|\nabla u|^{2} d y \leq \gamma \frac{1}{\left(r_{h}-r_{h+1}\right)^{2}} \sup _{A_{k_{h}, r_{h}}}\left(u-k_{h}\right)^{2} \leq \gamma \frac{2^{2 h+4}}{r^{2}} H^{2}\left|A_{k_{h}, r_{h}}\right| .
$$


We use inequality (3.4) from Lemma 3.4 to obtain that

$$
\left(k_{h+1}-k_{h}\right)\left|A_{k_{h+1}, r_{h+1}}\right|^{1-1 / n} \leq C \int_{A_{k_{h}, r_{h+1}}}|\nabla u| d y,
$$

where we choose $\theta_{1}$ small in order to obtain a uniform bound on the constant in the Sobolev inequality in (3.4). Now (3.6) and (3.7) give that

$$
\begin{aligned}
\frac{H}{2^{h+2}}\left|A_{k_{h+1}, r_{h+1}}\right|^{1-1 / n} & \leq C \int_{A_{k_{h}, r_{h+1}}}|\nabla u| d y \\
& \leq C\left(\int_{A_{k_{h}, r_{h+1}}}|\nabla u|^{2} d y\right)^{1 / 2}\left|A_{k_{h}, r_{h+1}}\right|^{1 / 2} \\
& \leq C \gamma^{1 / 2} 2^{h+2} \frac{H}{r}\left|A_{k_{h}, r_{h}}\right| .
\end{aligned}
$$

Thus, we may conclude that

$$
\left(\frac{\left|A_{k_{h+1}, r_{h+1}}\right|}{r^{n}}\right)^{1-1 / n} \leq C \gamma^{1 / 2} 2^{2 h+4} \frac{\left|A_{k_{h}, r_{h}}\right|}{r^{n}} .
$$

According to Lemma 4.7 in the monograph of Ladyzhenskaya and Ural'tseva 19 , p. 66], if $\theta_{1}$ is sufficiently small, then the recursion relation (3.8) implies that $\lim _{h \rightarrow \infty}\left|A_{k_{h}, r_{h}}\right| / r^{n}=0$.

We now give the main step in the proof of Hölder continuity of solutions of the mixed problem. Before stating the result, we introduce the notation $\operatorname{osc}_{E} u=$ $\sup _{E} u-\inf _{E} u$ for the oscillation of a real-valued function $u$ on a set $E$.

Lemma 3.5. Let $x \in \partial \Omega$ and assume that $0<r<r_{0}$. Let $u$ be a solution of $L u=0$ in $\Psi_{r}(x)$ and suppose that $u$ has zero data for the mixed problem on $\partial \Psi_{r}(x) \cap \partial \Omega$. Then there exists an integer s such that

$$
\underset{\Psi_{r}(x)}{\operatorname{OSc}} u \leq\left(1-2^{1-s}\right) \underset{\Psi_{4 r}(x)}{\operatorname{Osc}} u .
$$

Proof. Since osc $u=\operatorname{osc}(-u)$, it suffices to prove the lemma for either $u$ or $-u$. We will take advantage of this in the proof below. We define

$$
\begin{aligned}
M_{r} & =\sup \left\{u(x): x \in \Psi_{r}(x)\right\}, \\
m_{r} & =\inf \left\{u(x): x \in \Psi_{r}(x)\right\}, \\
\omega_{r} & =M_{r}-m_{r}=\underset{\Psi_{r}(x)}{\operatorname{osc}} u, \\
\bar{M}_{r} & =\frac{1}{2}\left(M_{r}+m_{r}\right) .
\end{aligned}
$$

In what follows, set $\omega=\omega_{4 r}$ and

$$
D_{t}=A_{M_{4 r}-\omega / 2^{t}, 2 r} \backslash A_{M_{4 r}-\omega / 2^{t+1}, 2 r}, \quad t=1,2, \ldots, s,
$$

where $s$ remains to be determined.

There are a few details that are different in the cases when $\Psi_{2 r}(x) \cap D \neq \emptyset$ and $\Psi_{2 r}(x) \cap D=\emptyset$, and we will point out the differences when they arise.

In the case when $\Psi_{2 r}(x) \cap D=\emptyset$, we may assume that

$$
\left|A_{\bar{M}_{4 r}, 2 r}\right| \leq \frac{1}{2}\left|\Psi_{2 r}(x)\right|
$$


for if condition (3.9) fails, we may replace $u$ by $-u$. We next use the inequality (3.4) with $k=M_{4 r}-\omega / 2^{t}$ and $\ell=M_{4 r}-\omega / 2^{t+1}$ to conclude that

$$
\frac{\omega}{2^{t+1}}\left|A_{M_{4 r}-\omega / 2^{t+1}, 2 r}\right|^{1-1 / n} \leq C \int_{D_{t}}|\nabla u| d y .
$$

Now since $D_{t} \subset A_{M_{4 r}-\omega / 2^{t}, 2 r}$ and $u \in \mathcal{B}\left(\Psi_{r}(x), \gamma\right)$, we have that

$\int_{D_{t}}|\nabla u|^{2} d y \leq \frac{\gamma}{4 r^{2}}\left|A_{M_{4 r}-\omega / 2^{t}, 4 r}\right| \sup _{A_{M_{4 r}-\omega / 2^{t}, 4 r}}\left(u-\left(M_{4 r}-\omega / 2^{t}\right)\right)^{2} \leq C\left(\frac{\omega}{2^{t}}\right)^{2} r^{n-2}$.

Thus, from (3.10) and the Cauchy-Schwarz inequality, we have that

$$
\left(\frac{\omega}{2^{t+1}}\right)^{2}\left|A_{M_{4 r}-\omega / 2^{t+1}, 2 r}\right|^{2-2 / n} \leq C\left|D_{t}\right|\left(\frac{\omega}{2^{t}}\right)^{2} r^{n-2} .
$$

If we sum (3.11) from $t=1, \ldots, s-3$, we conclude that

$$
(s-3)\left|A_{M_{4 r}-\omega / 2^{s-2}, 2 r}\right|^{2-2 / n} \leq C r^{n-2} \sum_{t=1}^{s-3}\left|D_{t}\right| \leq C_{0} r^{2 n-2} .
$$

Choose $s$ such that

with $\theta_{1}$ as in Lemma 3.3

$$
\left(\frac{C_{0}}{s-3}\right)^{n /(2 n-2)} \leq \theta_{1}
$$

Now let $k=M_{4 r}-\omega / 2^{s-2}$ and $H=\sup _{\Psi_{r}(x)}(u-k)=M_{r}-\left(M_{4 r}-\omega / 2^{s-2}\right)$. If $H>0$, we may apply Lemma 3.3 to obtain

$$
\sup _{\Psi_{r}(x)} u \leq k+H / 2 \leq M_{4 r}-\omega / 2^{s-2}+\frac{1}{2}\left(M_{r}-M_{4 r}-\omega / 2^{s-2}\right) .
$$

Simplifying, we find that

$$
M_{r} \leq M_{4 r}-\omega / 2^{s-1} .
$$

This inequality also follows easily if $H \leq 0$. It is immediate to see that $-m_{4 r} \geq$ $-m_{r}$, and if we recall that $\omega=\omega_{4 r}$, we may conclude that

$$
\omega_{r} \leq\left(1-1 / 2^{s-1}\right) \omega_{4 r} .
$$

Next we consider the case when $\Psi_{2 r}(x) \cap D \neq \emptyset$. In this situation we use the freedom to replace $u$ by $-u$ to impose the condition that $\bar{M}_{4 r} \geq 0$ and, as a result, (3.9) is not guaranteed to hold.

Since $M_{4 r}-\omega / 2^{t} \geq \bar{M}_{4 r} \geq 0$, we may use the Sobolev inequality (3.5) to obtain

$$
\frac{\omega}{2^{t+1}}\left|A_{M_{4 r}-\omega / 2^{t}, 2 r}\right|^{1-1 / n} \leq C \int_{D_{t}^{\prime}}|\nabla u| d y,
$$

where $D_{t}^{\prime}=A_{M_{4 r-\omega / 2^{t}, 4 r}} \backslash A_{M_{4 r-\omega / 2^{t+1,4 r}}}$. This replaces (3.10) in the argument that leads to (3.12). The rest of the argument goes throughout without change.

Proof of Theorem 3.1. The theorem follows immediately from Lemma 3.5.

We now return to working with only the Laplacian, as this will simplify the uniqueness argument below. We define a Green function with pole at $x$ for the mixed problem to be a function $G(x, \cdot) \in W^{1,1}(\Omega)$ which satisfies a) $G(x, \cdot) \in$ $W_{D}^{1,2}\left(\Omega \backslash B_{r}(x)\right)$ for each $r>0$ and b) if $\phi$ is in $C^{\infty}(\bar{\Omega})$ and vanishes on $D$, then

$$
\int_{\Omega} \nabla G(x, \cdot) \cdot \nabla \phi d y=-\phi(x) .
$$


The Green function is unique. If there are two candidates for the Green function with pole at $x, G_{1}(x, \cdot)$ and $G_{2}(x, \cdot)$, then $u=G_{1}(x, \cdot)-G_{2}(x, \cdot)$ will satisfy

$$
\int_{\Omega} \nabla u \cdot \nabla \phi d y=0
$$

for all $\phi \in C^{\infty}(\bar{\Omega})$ which vanish on $D$. From Weyl's lemma, $u$ is smooth in the interior of $\Omega$. Then the assumption that each $G_{i}(x, \cdot)$ lies in $W_{D}^{1,2}\left(\Omega \backslash B_{r}(x)\right)$ for each $r>0$ implies that $u$ is in $W_{D}^{1,2}(\Omega)$. Then $u$ is a weak solution of the mixed problem with zero data, and hence $u=0$. The properties of the Green function for the mixed problem that we will need in the sequel of this paper are summarized in the following lemma.

Lemma 3.6. Consider the mixed problem in a Lipschitz domain $\Omega$ with $D$ satisfying (2.2). Then there exists a Green function for the mixed problem which satisfies: 1) if $G_{x}(y)=G(x, y)$, then $G_{x} \in W_{D}^{1,2}\left(\Omega \backslash B_{r}(x)\right)$ for all $r>0$, 2) $\Delta G_{x}=\delta_{x}$, the Dirac $\delta$-measure at $x, 3)$ if $f_{N} \in W_{D}^{-1 / 2,2}(\partial \Omega)$, then the weak solution of the mixed problem with $f_{D}=0$ can be represented by

$$
u(x)=-\left\langle f_{N}, G_{x}\right\rangle,
$$

4) the Green function is Hölder continuous away from the pole and satisfies the estimates

and

$$
\begin{aligned}
&\left|G(x, y)-G\left(x, y^{\prime}\right)\right| \leq \frac{C\left|y-y^{\prime}\right|^{\beta}}{|x-y|^{n-2+\beta}},|x-y|>2\left|y-y^{\prime}\right|, \\
&|G(x, y)| \leq \frac{C}{|x-y|^{n-2}}, \quad n \geq 3,
\end{aligned}
$$

$$
|G(x, y)| \leq C(1+\log (d /|x-y|)), \quad n=2 .
$$

Above, the exponent $\beta$ is as in Theorem 3.1.

We give a detailed proof of this lemma when $n=2$. The proof for $n \geq 3$ may be obtained by adapting this argument or by a straightforward adaptation of the arguments in Grüter and Widman [12].

Proof. We begin with a result of Kenig and Ni [17] who established the existence of global fundamental solutions in two dimensions. Suppose that $L=\operatorname{div} A \nabla$ is an elliptic operator in two dimensions with bounded, measurable, and symmetric coefficients. Then there exists a fundamental solution $\Gamma(x, y)$ which satisfies $\Gamma(x, \cdot) \in W_{l o c}^{1,2}\left(\mathbf{R}^{2} \backslash\{x\}\right), \Gamma(x, \cdot) \in W_{l o c}^{1,1}\left(\mathbf{R}^{2}\right)$, and we have

$$
\int_{\mathbf{R}^{2}} A \nabla \Gamma(x, \cdot) \cdot \nabla \phi d y=-\phi(x), \quad \text { for all } \phi \in C_{c}^{\infty}\left(\mathbf{R}^{2}\right) .
$$

Chanillo and Li [4, Corollary 1.1] observe that the free space fundamental solution lies in $B M O\left(\mathbf{R}^{2}\right)$, and thus if $\bar{\Gamma}=f_{B_{s}(z)} \Gamma(x, y) d y$, we have

$$
f_{B_{s}(z)}(\Gamma(x, y)-\bar{\Gamma})^{2} d y \leq C,
$$

where the constant depends only on the ellipticity constant for the operator $L$ and the bounds for the coefficients.

Next we recall a result of Dahlberg and Kenig [9, p. 447], where if $\Omega^{\prime}=\left\{\left(x_{1}, x_{2}\right)\right.$ : $\left.x_{2}>\psi\left(x_{1}\right)\right\}$ is the domain which lies above the graph of a single Lipschitz function, 
then there exists a Green function for the Neumann problem in $\Omega^{\prime}$ which can be constructed by the method by reflection. We briefly recall the construction of this Green function. Define $R$, a reflection in $\partial \Omega^{\prime}$, by $R(x)=\left(x_{1}, 2 \psi\left(x_{1}\right)-x_{2}\right)$ and construct an operator $L=\operatorname{div} A \nabla$ on $\mathbf{R}^{2}$ so that $L(u \circ R)=0$ in $\mathbf{R}^{2} \backslash \bar{\Omega}^{\prime}$ if and only if $\Delta u=0$ in $\Omega^{\prime}$ and $L=\Delta$ in $\Omega^{\prime}$. Let $\Gamma$ be the fundamental solution for this operator in $\mathbf{R}^{2}$ and define

$$
\mathcal{N}(x, y)=\Gamma(x, y)+\Gamma(R x, y) .
$$

We have that $\mathcal{N}$ is a fundamental solution in $\Omega^{\prime}$ with zero Neumann data on $\partial \Omega^{\prime}$. More precisely, we have the weak formulation

$$
\int_{\Omega^{\prime}} \nabla \mathcal{N}(x, \cdot) \cdot \nabla \phi d y=-\phi(x), \quad \text { for all } \phi \in C_{c}^{\infty}(\bar{\Omega}) .
$$

We give a detailed proof of the construction of the Green function for the mixed problem $G(x, \cdot)$ when $x$ is near the boundary and hence lies in some coordinate cylinder. When $x$ is far from the boundary, the construction of the Green function is simpler, and we omit the details. Fix $x$ in our original domain $\Omega$ and assume that $x$ lies in a coordinate cylinder $Z_{r}\left(x_{0}\right)$ with $Z_{4 r}\left(x_{0}\right)$ also a coordinate cylinder. Let $s=\min (\operatorname{dist}(x, D), r)$ and then construct a cutoff function $\eta$ which is one on $B_{s / 2}(x)$ and zero outside $B_{s}(x)$. We let $\mathcal{N}$ be the Green function for the Neumann problem in a graph domain $\Omega^{\prime}$ that satisfies $\Omega \cap Z_{4 r}=\Omega^{\prime} \cap Z_{4 r}$. Since $\Gamma$ and hence $\mathcal{N}$ lie in $B M O\left(\mathbf{R}^{2}\right)$, we may choose a constant $\overline{\mathcal{N}}$ so that

$$
f_{\Psi_{s}(x)}(\mathcal{N}(x, y)-\overline{\mathcal{N}})^{2} d y \leq C,
$$

where the bound $C$ depends only on the Lipschitz constant $M$.

We will look for the Green function for the mixed problem in the form

$$
G(x, y)=\eta(y)(\mathcal{N}(x, y)-\overline{\mathcal{N}})+u(y) .
$$

We will show that the function $u$ lies in $W_{D}^{1,2}(\Omega)$ with a bound depending only on the Lipschitz constant $M$ and the constant in (3.2).

If $G$ is to be a Green function, we need $u$ to satisfy

$$
\int_{\Omega}(\eta \nabla \mathcal{N}(x, \cdot)+(\mathcal{N}(x, \cdot)-\overline{\mathcal{N}}) \nabla \eta+\nabla u) \cdot \nabla \phi d y=-\phi(x)
$$

for all functions $\phi$ which lie in $C^{\infty}(\bar{\Omega})$ and vanish on $D$. We write $\eta \nabla \phi=\nabla(\eta \phi)-$ $\phi \nabla \eta$ and get

$\int_{\Omega} \nabla \mathcal{N}(x, \cdot) \cdot \nabla(\eta \phi)-\phi \nabla \mathcal{N}(x, \cdot) \cdot \nabla \eta+(\mathcal{N}(x, \cdot)-\overline{\mathcal{N}}) \nabla \eta \cdot \nabla \phi+\nabla u \cdot \nabla \phi d y=-\phi(x)$.

Since $\mathcal{N}$ is a Green function for the Neumann problem, we may use (3.14) to simplify the previous equation and obtain

$$
\int_{\Omega} \nabla u \cdot \nabla \phi d y=\int_{\Omega} \phi \nabla \mathcal{N}(x, \cdot) \cdot \nabla \eta-(\mathcal{N}(x, \cdot)-\overline{\mathcal{N}}) \nabla \eta \cdot \nabla \phi d y .
$$

Let $F(\phi)$ denote the right-hand side of (3.16). We claim that $|F(\phi)| \leq C\|\phi\|_{W_{D}^{1,2}(\Omega)}$, where the constant depends only on the constant $M$ and the constant in the coercivity estimate (3.2). From the claim and basic results about Hilbert spaces, it follows that there exists a solution $u$ to (13.16) and this solution satisfies $\|u\|_{W_{D}^{1,2}(\Omega)} \leq C$. 
We now turn to the proof of the claim. To estimate the first term of (3.16), we begin with an application of the Cauchy-Schwarz inequality

$$
\left|\int_{\Omega} \phi \nabla \mathcal{N}(x, \cdot) \cdot \nabla \eta d y\right| \leq C\left(\int_{\Psi_{s}(x) \backslash \Psi_{s / 2}(x)}|\nabla \mathcal{N}(x, \cdot)|^{2} d y\right)^{\frac{1}{2}}\left(f_{\Psi_{s}(x)} \phi^{2} d y\right)^{\frac{1}{2}} .
$$

Using the Caccioppoli inequality and that $\mathcal{N}$ is in $B M O\left(\mathbf{R}^{2}\right)$ (see (3.15)), we may conclude

$$
\left(\int_{\Psi_{s}(x) \backslash \Psi_{s / 2}(x)}|\nabla \mathcal{N}(x, \cdot)|^{2} d y\right)^{1 / 2} \leq C,
$$

where the constant depends only on the Lipschitz constant for $\Omega$. Thanks to the choice of $s$, we may use that $\phi$ vanishes on $D$ and (2.2) to obtain the Poincaré inequality

$$
f_{\Psi_{s}(x)} \phi^{2} d y \leq C \int_{\Psi_{C s}(x)}|\nabla \phi|^{2} d y
$$

See Section 3 of Ott and Brown [26] for details.

Thus we obtain the estimate

$$
\left|\int_{\Omega} \phi \nabla \mathcal{N}(x, \cdot) \cdot \nabla \eta d y\right| \leq C\|\nabla \phi\|_{L^{2}(\Omega)} .
$$

The estimate for the other term,

$$
\left|\int_{\Omega}(\mathcal{N}(x, \cdot)-\overline{\mathcal{N}}) \nabla \eta \cdot \nabla \phi d y\right| \leq C\|\nabla \phi\|_{L^{2}(\Omega)},
$$

follows from the Cauchy Schwarz inequality since $\mathcal{N}(x, \cdot)$ is in $B M O\left(\mathbf{R}^{2}\right)$.

Next we recall that if $u$ is in $W^{1,2}(\Omega), x \in \Omega$, and $r>0$, we may find a constant $\bar{u}$ such that we have the Poincaré inequality

$$
f_{\Psi_{r}(x)}(u-\bar{u})^{2} d y \leq C\|\nabla u\|_{L^{2}(\Omega)}^{2} .
$$

In other words, $u$ lies in $B M O(\Omega)$. Since we also have that $\mathcal{N}$ is in $B M O\left(\mathbf{R}^{2}\right)$, we may conclude that $G$ lies in $B M O(\Omega)$.

Now we turn to the estimates in parts 3) and 4) of Lemma 3.6. First, recall that if $v$ is in $B M O(\Omega)$ and $\Psi_{r}(x) \cap \Psi_{2 r}\left(x^{\prime}\right) \neq \emptyset$, then we have

$$
\left|f_{\Psi_{r}(x)} v d y-f_{\Psi_{2 r}\left(x^{\prime}\right)} v d y\right| \leq\|v\|_{*},
$$

where $\|v\|_{*}$ is the $B M O$ norm of $v$. Using this, an iteration argument and the local boundedness estimate (3.3), we obtain the pointwise upper bound,

$$
|G(x, y)| \leq C(1+\log (d /|x-y|)),
$$

where $d$ is the diameter of $\Omega$ and the constant $C$ depends on $\Omega$. Next we show that the Green function is Hölder continuous. Let $v$ be a solution of $L v=0$ in $\Psi_{r}(x)$ with zero data for the mixed problem on $\partial \Omega \cap \partial \Psi_{r}(x)$. From the local boundedness result (3.3) and the estimate for Hölder continuity in Theorem 3.1, for any constant $\bar{v}$ we have

$$
\left|v(y)-v\left(y^{\prime}\right)\right| \leq C\left(\left|y-y^{\prime}\right| / r\right)^{\alpha} f_{\Psi_{r}(x)}|v-\bar{v}| d y, \quad y, y^{\prime} \in \Psi_{r / 2}(x) .
$$


Since $G$ is in $B M O(\Omega)$, the Hölder estimate for $G$ in part 4) of Lemma 3.6 follows by applying the above observation to $G(x, \cdot)$ on a ball centered at $y$ with radius $r=|x-y|$.

Next, we claim that if $f_{N} \in W_{D}^{-1 / 2,2}(\partial \Omega)$ and $u$ is the weak solution of the mixed problem with Neumann data $f_{N}$ and zero Dirichlet data, then we have the representation formula in part 3) of Lemma 3.6.

$$
u(x)=-\left\langle f_{N}, G_{x}\right\rangle .
$$

Here, $G_{x}=G(x, \cdot)$ and $\langle\cdot, \cdot\rangle$ denotes the duality pairing between $W_{D}^{-1 / 2,2}(\partial \Omega)$ and $W_{D}^{1 / 2,2}(\partial \Omega)$. To begin the proof of (3.17), consider $\int_{\Omega} \nabla G(x, \cdot) \cdot \nabla u d y$. Fix $x \in \Omega$, let $r=\operatorname{dist}(x, \partial \Omega) / 2$, and let $\eta$ be a cutoff function with $\eta=1$ on $B_{r / 2}(x)$ and $\eta=0$ outside $B_{r}(x)$. We may approximate $\eta G(x, \cdot)$ in $W^{1,1}(\Omega)$ by a sequence of smooth functions and use that $u$ is harmonic in $B_{r}(x)$ to conclude that $\int_{\Omega} \nabla(\eta G(x, \cdot))$. $\nabla u d y=0$. Since $(1-\eta) G(x, \cdot)$ lies in $W_{D}^{1,2}(\Omega)$, we may use that $u$ is a weak solution of the mixed problem to conclude that $\int_{\Omega} \nabla((1-\eta) G(x, \cdot)) \cdot \nabla u d y=\left\langle f_{N}, G_{x}\right\rangle$. Combining these observations gives

$$
\int_{\Omega} \nabla G(x, \cdot) \cdot \nabla u d y=\left\langle f_{N}, G_{x}\right\rangle .
$$

We now reverse the roles of $G$ and $u$. With $\eta$ as above, write $u=\eta u+(1-\eta) u$. As $u$ is harmonic and hence smooth in the interior of $\Omega$, there exists a sequence of smooth, compactly supported functions which converge to $\eta u$ in $W^{1, \infty}(\Omega)$. We may use this sequence and (3.13) to obtain $\int_{\Omega} \nabla G \cdot \nabla(\eta u) d y=-u(x)$. As $u$ lies in $W_{D}^{1,2}(\Omega)$ we may find a sequence $\left\{u_{k}\right\}$ of smooth functions which vanish on $D$ and which converge in $W^{1,2}(\Omega)$ to $u$. Approximating $(1-\eta) u$ by $(1-\eta) u_{k}$ and using (3.13) gives $\int_{\Omega} \nabla G(x, \cdot) \cdot \nabla((1-\eta) u) d y=0$. Combining these observations implies

$$
\int_{\Omega} \nabla G(x, \cdot) \cdot \nabla u d y=-u(x) .
$$

From (3.18) and (3.19), we obtain (3.17).

The next two lemmas establish higher integrability of the gradient of weak solutions to the mixed problem. The proofs of these lemmas appear in Ott and Brown [26. Section 3]. The key ingredients of the proofs appearing in the aforementioned paper are Poincaré inequalities, and these Poincaré inequalities continue to hold true in the current setting due to condition (2.2) on $D$. A similar estimate is obtained for the mixed problem by Gröger 11 using the method of N. Meyers 22 . However, Gröger's method requires more restrictions on the boundary between $D$ and $N$.

Lemma 3.7. Let $\Omega$ and $D$ satisfy (1.2) and (2.2). Let $x \in \Omega$ and let $r$ satisfy $0<r<r_{0}$. Let $u$ be a weak solution of the mixed problem for a divergence form elliptic operator with zero Dirichlet data and Neumann data $f_{N} \in L^{q}(N)$. Then $u$ satisfies the estimate

$$
\left(f_{\Psi_{r}(x)}|\nabla u|^{2} d y\right)^{1 / 2} \leq C\left[f_{\Psi_{C r}(x)}|\nabla u| d y+\left(\frac{1}{r^{n-1}} \int_{\Delta_{C r}(x)}\left|f_{N}\right|^{q} d \sigma\right)^{1 / q}\right] .
$$

Above, $q=2$ if $n=2$ and $q=2(n-1) /(n-2)$ for $n \geq 3$. The constant $C$ depends on $M$ and $n$. 
Lemma 3.8. Let $\Omega$ and $D$ satisfy (1.2) and (2.2). Let $x \in \Omega$ and let $r$ satisfy $0<r<r_{0}$. Let $u$ be a weak solution of the mixed problem with zero Dirichlet data and Neumann data $f_{N} \in L^{q}(N)$ which is supported in $N \cap \Delta_{r}(x)$, with $q$ as in Lemma 3.7. Then there exists $q_{0}=q_{0}(M, n)>2$ such that for $t$ in the range $2 \leq t<q_{0}$ when $n \geq 3$ and $t$ in the range $2<t<q_{0}$ when $n=2$, u satisfies the estimate

$$
\begin{aligned}
& \left(f_{\Psi_{r}(x)}|\nabla u|^{t} d y\right)^{1 / t} \\
& \leq C\left[f_{\Psi_{C r}(x)}|\nabla u| d y+\left(\frac{1}{r^{n-1}} \int_{\Delta_{C r}(x)}\left|f_{N}\right|^{t(n-1) / n} d \sigma\right)^{n /(t(n-1))}\right] .
\end{aligned}
$$

The constant above depends on $M$ and $n$.

\section{Estimates For Solutions With ATOMiC Data}

In this section we consider the mixed problem with Neumann data an atom for $N$ and zero Dirichlet data. We estimate the decay of the solution of this mixed problem as we move away from the support of the atom by taking $L^{p}$-norms of the solution in dyadic rings around the support of the atom. Thus, given a surface ball $\Delta_{r}(x)$, we define $\Sigma_{k}=\Delta_{2^{k} r}(x) \backslash \Delta_{2^{k-1} r}(x)$ and $S_{k}=\Psi_{2^{k} r}(x) \backslash \Psi_{2^{k-1} r}(x)$.

Theorem 4.1. Let $\Omega$ and $D$ satisfy (1.2) and (1.4). Fix $x \in \partial \Omega$ and let $r$ satisfy $0<r<r_{0}$. Let $u$ be a weak solution of the mixed problem (1.1) with Neumann data $f_{N}=a$ an atom for $N$ which is supported in $\Delta_{r}(x)$ and zero Dirichlet data. Let $q_{0}>2$ be as in Lemma 3.8 and let $\Lambda$ satisfy (1.3) with $\epsilon$ such that $0 \leq \epsilon<$ $\left(q_{0}-2\right) /\left(q_{0}-1\right)$. Then for $1<p<q_{0}((1-\epsilon) /(2-\epsilon))$, the following estimates hold:

$$
\left(\int_{\Delta_{8 r}(x)}|\nabla u|^{p} d \sigma\right)^{1 / p} \leq C \sigma\left(\Delta_{r}(x)\right)^{-1 / p^{\prime}},
$$

and for $k \geq 4$,

$$
\left(\int_{\Sigma_{k}}|\nabla u|^{p} d \sigma\right)^{1 / p} \leq C 2^{-\beta k} \sigma\left(\Sigma_{k}\right)^{-1 / p^{\prime}} .
$$

Here, $\beta$ is as in Lemma 3.6 and the constants in the estimates (4.1) and (4.2) depend on $p$ and the global character of the domain.

In order to prove Theorem 4.1 we need a series of lemmas, some of which require that we work in a subdomain of $\Omega$ which is also contained in a coordinate cylinder. For $x \in \partial \Omega$ and $r$ satisfying $0<r<r_{0}$, let $\Omega_{r}(x)=Z_{r}(x) \cap \Omega$, where $Z_{r}(x)$ is a coordinate cylinder as defined in Section 2. The sets $\Omega_{r}(x)$ are star-shaped Lipschitz domains, and for this reason they are preferable to the sets $\Psi_{r}(x)$.

The following lemmas rely on a Whitney decomposition of $\partial \Omega \backslash \Lambda$. For simplicity, we use surface cubes rather than the surface balls used up to this point. A surface cube is the image of a cube in $\mathbf{R}^{n-1}$ under the mapping $x^{\prime} \rightarrow\left(x^{\prime}, \phi\left(x^{\prime}\right)\right)$. Then we write $\partial \Omega=\Lambda \cup\left(\bigcup_{j} Q_{j}\right)$, where the collection of surface cubes $\left\{Q_{j}\right\}$ has the following three properties: 1) for each $j$, either $Q_{j} \subset D$ or $Q_{j} \subset(N \backslash \Lambda)$, 2) there exist constants $c^{\prime}$ and $c^{\prime \prime}$, with $c^{\prime \prime}$ as small as we like, such that for each $x \in Q$ and each $\left.j, c^{\prime} \delta(x)<\operatorname{diam}\left(Q_{j}\right)<c^{\prime \prime} \delta(x), 3\right)$ if $T\left(Q_{j}\right)=\left\{x \in \bar{\Omega}: \operatorname{dist}(x, \partial \Omega)<\operatorname{diam}\left(Q_{j}\right)\right\}$, 
then provided that the constants in the previous condition are sufficiently small, the sets $\left\{T\left(Q_{j}\right)\right\}$ have bounded overlaps and thus

$$
\sum_{j} \chi_{T\left(Q_{j}\right)} \leq C(n, M)
$$

We now begin the series of lemmas. The first two lemmas give a local version of boundary regularity for the Dirichlet and Neumann problems for the Laplacian, and they require only that $\Omega$ be a Lipschitz domain. The proofs of the next two lemmas appear in the previous work of Ott and Brown [26, Section 4].

Lemma 4.2. Let $\Omega$ be a Lipschitz domain, let $x \in \partial \Omega$, and assume that $r$ satisfies $0<r<r_{0}$. Let $u$ be a harmonic function in $\Omega_{4 r}(x)$. If $\nabla u \in L^{2}\left(\Omega_{4 r}(x)\right)$ and $\partial u / \partial \nu \in L^{2}\left(\partial \Omega \cap \partial \Omega_{4 r}\right)$, then $\nabla u \in L^{2}\left(\Delta_{r}(x)\right)$ and

$$
\int_{\Delta_{r}(x)}\left((\nabla u)_{r}^{*}\right)^{2} d \sigma \leq C\left(\int_{\partial \Omega \cap \partial \Omega_{4 r}(x)}\left|\frac{\partial u}{\partial \nu}\right|^{2} d \sigma+\frac{1}{r} \int_{\Omega_{4 r}(x)}|\nabla u|^{2} d y\right) .
$$

The constant $C$ depends on $M$ and the dimension $n$.

Lemma 4.3. Let $\Omega$ be a Lipschitz domain, let $x \in \partial \Omega$ and let $r$ be such that $0<r<r_{0}$. Let $u$ be a harmonic function in $\Omega_{4 r}(x)$. If $\nabla u \in L^{2}\left(\Omega_{4 r}(x)\right)$ and $\nabla_{t} u \in L^{2}\left(\partial \Omega \cap \partial \Omega_{4 r}(x)\right)$, then $\nabla u \in L^{2}\left(\Delta_{r}(x)\right)$ and

$$
\int_{\Delta_{r}(x)}\left((\nabla u)_{r}^{*}\right)^{2} d \sigma \leq C\left(\int_{\partial \Omega \cap \partial \Omega_{4 r}(x)}\left|\nabla_{t} u\right|^{2} d \sigma+\frac{1}{r} \int_{\Omega_{4 r}(x)}|\nabla u|^{2} d y\right) .
$$

The constant $C$ depends on $M$ and the dimension $n$.

The next lemma also appears in Ott and Brown [26, Section 4]. For the sake of completeness we sketch the proof again in this paper.

Lemma 4.4. Let $\Omega$ and $D$ satisfy (1.2), (1.3), and (1.4). Let $u$ be a weak solution of the mixed problem with Neumann data $f_{N} \in L^{2}(N)$ and zero Dirichlet data. Let $\rho \in \mathbf{R}, x \in \partial \Omega$, and $0<r<r_{0}$, and assume that for some $A>0, \delta(x) \leq A r$. Then it follows that

$$
\int_{\Delta_{r}(x)}\left((\nabla u)_{c \delta}^{*}\right)^{2} \delta^{1-\rho} d \sigma \leq C\left(\int_{\Delta_{2 r}(x)}\left|f_{N}\right|^{2} \delta^{1-\rho} d \sigma+\int_{\Psi_{2 r}(x)}|\nabla u|^{2} \delta^{-\rho} d y\right)
$$

for constants $c$ and $C$ which depend only on $M, n, A$ and $\rho$.

Proof. When $\Delta_{r}(x)$ is close to $\Lambda$, using the Whitney decomposition constructed above and the estimates of Lemma 4.2 and 4.3 , we have

$$
\int_{Q_{j}}\left((\nabla u)_{r_{j}}^{*}\right)^{2} d \sigma \leq C\left(\int_{2 Q_{j} \cap N}\left|\frac{\partial u}{\partial \nu}\right|^{2} d \sigma+\frac{1}{r_{j}} \int_{T\left(Q_{j}\right)}|\nabla u|^{2} d y\right) .
$$

To finish the proof of the lemma, we multiply (4.3) by $r_{j}^{-\rho}$, recall that $r_{j} \approx \delta(x)$ for all $x \in T\left(Q_{j}\right)$, sum on the $Q_{j}$ that intersect $\Delta_{r}(x)$, and use that the family $\left\{T\left(Q_{j}\right)\right\}$ has bounded overlaps.

The next result is another reverse Hölder inequality, this time at the boundary. While at first glance the result below may not resemble a reverse Hölder inequality, in future applications in this paper $f_{N}=0$ or a constant. 
Theorem 4.5. Let $\Omega$ and $D$ satisfy (1.2) and (1.4). Let $q_{0}>2$ be as in Lemma 3.8 and let $\Lambda$ satisfy (1.3) with $0 \leq \epsilon<\left(q_{0}-2\right) /\left(q_{0}-1\right)$. Let $u$ be the weak solution of the mixed problem with Neumann data $f_{N} \in L^{2}(N)$ and zero Dirichlet data. Fix $p$ such that $1<p<q_{0}(1-\epsilon) /(2-\epsilon)$. For $x \in \partial \Omega$ and $r$ satisfying $0<r<r_{0}$,

$$
\left(f_{\Delta_{r}(x)}|\nabla u|^{p} d \sigma\right)^{1 / p} \leq C\left(f_{\Psi_{2 r}(x)}|\nabla u| d y+r^{(1-n) / 2}\left\|f_{N}\right\|_{L^{2}\left(\Delta_{2 r}(x) \cap N\right)}\right)
$$

Above, the constant $C$ depends on $M$, the dimension $n$, and $p$.

Proof. Fix $x \in \partial \Omega$ and $0<r<r_{0}$. We claim that

$$
\left(f_{\Delta_{4 r}(x)}|\nabla u|^{p} d \sigma\right)^{1 / p} \leq C\left(f_{\Psi_{16 r}(x)}|\nabla u| d y+r^{(1-n) / 2}\left\|f_{N}\right\|_{L^{2}\left(\Delta_{32 r}(x) \cap N\right)}\right) .
$$

We will separate the proof into two cases: a) $\delta(x) \leq 8 r \sqrt{1+M^{2}}$, and b) $\delta(x)>$ $8 r \sqrt{1+M^{2}}$. Starting with case a), choose $\rho$ satisfying $2-\frac{2}{p}(1-\epsilon)-\epsilon<\rho<$ $2-\frac{4}{q_{0}}(1-\epsilon)-\epsilon$ (the assumption that $0 \leq \epsilon<\left(q_{0}-2\right) /\left(q_{0}-1\right)$ ensures that this is a non-empty interval). Apply Hölder's inequality with the exponents $2 / p$ and $2 /(2-p)$ to get

$$
\begin{gathered}
\left(\int_{\Delta_{4 r}(x)}|\nabla u|^{p} d \sigma\right)^{1 / p} \\
\leq\left(\int_{\Delta_{4 r}(x)}|\nabla u|^{2} \delta^{1-\rho} d \sigma\right)^{\frac{1}{2}}\left(\int_{\Delta_{4 r}(x)} \delta^{(\rho-1) \frac{p}{2-p}} d \sigma\right)^{\frac{1}{p}-\frac{1}{2}} \\
\leq C r^{(n-1)\left(\frac{1}{p}-\frac{1}{2}\right)+\frac{\rho-1}{2}}\left(\int_{\Delta_{4 r}(x)}|\nabla u|^{2} \delta^{1-\rho} d \sigma\right)^{1 / 2}
\end{gathered}
$$

where we have used that $(\rho-1) p /(2-p)<-(1-\epsilon)$ or $\rho>2-\frac{2}{p}(1-\epsilon)-\epsilon$, and Lemma 2.4 to ensure that the integral of $\delta^{(\rho-1)(p /(2-p))}$ is finite. Next, we use Lemma 4.6 and our hypothesis that $\delta(x) \leq 8 r \sqrt{1+M^{2}}$ to obtain

$$
\begin{gathered}
\left(\int_{\Delta_{4 r}(x)}|\nabla u|^{2} \delta^{1-\rho} d \sigma\right)^{1 / 2} \\
\leq C\left[\left(\int_{\Psi_{8 r}(x)}|\nabla u|^{2} \delta^{-\rho} d y\right)^{1 / 2}+\left(\int_{\Delta_{8 r}(x) \cap N}\left|f_{N}\right|^{2} \delta^{1-\rho} d \sigma\right)^{1 / 2}\right] \\
\leq C\left[\left(\int_{\Psi_{8 r}(x)}|\nabla u|^{2} \delta^{-\rho} d y\right)^{1 / 2}+r^{\frac{n-\rho}{2}}\left\|f_{N}\right\|_{L^{2}\left(N \cap \Delta_{8 r}(x)\right)}\right] .
\end{gathered}
$$

To estimate the term $\left(\int_{\Psi_{8 r}(x)}|\nabla u|^{2} \delta^{-\rho} d y\right)^{1 / 2}$, choose $q>2$ such that $q<$ $\min \left\{q_{0}, 2 n /(n-1)\right\}$ and apply Hölder's inequality again with exponents $q / 2$ and 
$q /(q-2)$ to conclude

$$
\begin{gathered}
\left(\int_{\Psi_{8 r}(x)}|\nabla u|^{2} \delta^{-\rho} d y\right)^{1 / 2} \\
\leq\left(\int_{\Psi_{8 r}(x)}|\nabla u|^{q} d y\right)^{1 / q}\left(\int_{\Psi_{8 r}(x)} \delta^{-\rho \frac{q}{q-2}} d y\right)^{\frac{1}{2}-\frac{1}{q}} .
\end{gathered}
$$

We invoke Lemma 3.8 again, requiring that $q \in\left(2, q_{0}\right)$, and get the following bound:

$$
\begin{aligned}
& \left(\int_{\Psi_{8 r}(x)}|\nabla u|^{2} \delta^{-\rho} d y\right)^{1 / 2} \\
& \quad \leq C r^{\frac{n-\rho}{2}}\left[f_{\Psi_{16 r}(x)}|\nabla u| d y+\left(\frac{1}{r^{n-1}} \int_{\Delta_{16 r}(x) \cap N}\left|f_{N}\right|^{\frac{q(n-1)}{n}} d \sigma\right)^{\frac{n}{q(n-1)}}\right] .
\end{aligned}
$$

By our choice of $q$, we can apply Hölder's inequality with exponent $2 n /(q(n-1))$ to the boundary term in (4.8) to obtain

$$
\begin{aligned}
&\left(\frac{1}{r^{n-1}} \int_{\Delta_{16 r}(x) \cap N}\left|f_{N}\right|^{\frac{q(n-1)}{n}} d \sigma\right)^{\frac{n}{q(n-1)}} \\
& \leq C\left(\frac{1}{r^{n-1}} \int_{\Delta_{16 r}(x) \cap N}\left|f_{N}\right|^{2} d \sigma\right)^{1 / 2} .
\end{aligned}
$$

Combining equations (4.6), (4.7), (4.8), and (4.9) we conclude that

$$
\left(\int_{\Delta_{4 r}(x)}|\nabla u|^{p} d \sigma\right)^{\frac{1}{p}} \leq C r^{\frac{n-1}{p}}\left(f_{\Psi_{16 r}(x)}|\nabla u| d y+r^{(1-n) / 2}\left\|f_{N}\right\|_{L^{2}\left(\Delta_{16 r}(x) \cap N\right)}\right),
$$

which gives claim (4.5).

Now we prove claim (4.5) under the condition in case b). As with case a), we begin with an application of Hölder's inequality:

$$
\left(\int_{\Delta_{4 r}(x)}|\nabla u|^{p} d \sigma\right)^{1 / p} \leq C r^{(n-1)(1 / p-1 / 2)}\left(\int_{\Delta_{4 r}(x)}|\nabla u|^{2} d \sigma\right)^{1 / 2} .
$$

Use that $\delta(x)>8 r \sqrt{1+M^{2}}$ and Lemma 2.1 to conclude that either $\Delta_{8 r}(x) \subset N$ or $\Delta_{8 r}(x) \subset D$. Then we may appeal to Lemma 4.2 or Lemma 4.3 to obtain that

$$
\int_{\Delta_{4 r}(x)}|\nabla u|^{2} d \sigma \leq C\left(\int_{\Delta_{8 r}(x) \cap N}\left|f_{N}\right|^{2} d \sigma+\frac{1}{r} \int_{\Psi_{8 r}(x)}|\nabla u|^{2} d y\right) .
$$

From this point we must distinguish between $n=2$ and $n \geq 3$. First, let $n \geq 3$. Then Lemma 3.8 and Hölder's inequality give that

$$
\left(f_{\Psi_{8 r}(x)}|\nabla u|^{2} d y\right)^{\frac{1}{2}} \leq C\left[f_{\Psi_{16 r}(x)}|\nabla u| d y+\left(\int_{\Delta_{16 r}(x) \cap N}\left|f_{N}\right|^{\frac{2(n-1)}{n}} d \sigma\right)^{\frac{n}{2(n-1)}}\right] .
$$


Combining the last three displayed inequalities, we obtain

$$
\left(\int_{\Delta_{4 r}(x)}|\nabla u|^{p} d \sigma\right)^{\frac{1}{p}} \leq C r^{\frac{n-1}{p}}\left(f_{\Psi_{16 r}(x)}|\nabla u| d y+r^{(1-n) / 2}\left\|f_{N}\right\|_{L^{2}\left(\Delta_{16 r}(x) \cap N\right)}\right),
$$

which immediately leads to claim (4.5). In the case $n=2$, we need an additional application of Hölder's inequality. Choose $t$ satisfying $2<t<q_{0}$. Then $\left(f_{\Psi_{16 r}(x)}|\nabla u|^{2} d y\right)^{1 / 2} \leq C\left(f_{\Psi_{16 r}(x)}|\nabla u|^{t} d y\right)^{1 / t}$, and from this point we can now apply Lemma 3.8 to get the average of the square of the Neumann data. This gives (4.5).

Once (4.5) is established, an elementary covering argument leads to the desired estimate.

The final result we require before proving Theorem 4.1 is an energy estimate. The proof appears in Ott and Brown [26, Section 4].

Lemma 4.6. Let $u$ be a weak solution of the mixed problem with Neumann data $f_{N}$. For $n \geq 3$, let $f_{N} \in L^{p}(N)$ with $p=(2 n-2) / n$. Then the following estimate holds:

$$
\int_{\Omega}|\nabla u|^{2} d y \leq C\left\|f_{N}\right\|_{L^{p}(N)}^{2}
$$

If $n=2$, let $f_{N} \in H^{1}(N)$, and then the following estimate holds:

$$
\int_{\Omega}|\nabla u|^{2} d y \leq C\left\|f_{N}\right\|_{H^{1}(N)}^{2} .
$$

In both cases, the constant $C$ depends on the global character of $\Omega$.

We are now equipped to prove Theorem 4.1.

Proof of Theorem 4.1. Fix $x \in \partial \Omega$ and let $r$ satisfy $0<r<r_{0}$. The first step is to obtain an estimate for the gradient of the solution $u$ near the support of the atom. Estimate (4.1) follows immediately from Theorem 4.5. Lemma 4.6, and the normalization of the atom.

The next step is to estimate $\int_{\Sigma_{k}}|\nabla u|^{p} d \sigma$ for $k \geq 4$. We begin by proving that the solution $u$ satisfies the upper bound

$$
|u(y)| \leq \frac{C r^{\beta}}{|x-y|^{n-2+\beta}}, \quad|x-y|>2 r,
$$

where $\beta$ is as in Lemma 3.6. To establish (4.10), we use the representation formula in part 3) of Lemma 3.6 and claim that there exists $\bar{x}$ in $\Delta_{r}(x)$ such that

$$
u(y)=-\int_{\Delta_{r}(x) \cap N} a(z)(G(y, z)-G(y, \bar{x})) d \sigma .
$$

If $\Delta_{r}(x) \subset N$, then let $\bar{x}=x$ and use that $a$ has mean value zero to obtain the estimate (4.10). If $\Delta_{r}(x) \cap D \neq \emptyset$, then choose $\bar{x} \in D \cap \Delta_{r}(x)$ and use that $G(y, \cdot)$ vanishes on $D$. Now estimate (4.10) follows easily from the normalization of the atom and the estimates for the Green function in part 4) of Lemma 3.6.

The remainder of the proof of estimate (4.2) follows from Theorem 4.5] and estimate (4.10). The constant in the estimate will depend on $p, M$, the dimension $n$, and the collection of coordinate cylinders. 
Next we prove that the non-tangential maximal function of a weak solution lies in $L^{1}(\partial \Omega)$ when the Neumann data is given by an atom. We introduce the following notation to be used in the proof. Let

$$
\mathcal{C}_{t}=\{y: t<\delta(y)<2 t\} \cap \Omega \quad \text { and } \quad C_{t}=\{y: t<\delta(y)<2 t\} \cap \partial \Omega .
$$

Theorem 4.7. Let $\Omega$ and $D$ satisfy (1.2) and (1.4). Let $\Lambda$ satisfy (1.3) with $0 \leq \epsilon<\left(q_{0}-2\right) /\left(q_{0}-1\right)$, with $q_{0}>2$ from Lemma 3.8. If $f_{N} \in H^{1}(N)$, then there exists a solution $u$ of the $L^{1}$-mixed problem (1.1) with Neumann data $f_{N}$ and zero Dirichlet data. This solution satisfies

$$
\left\|(\nabla u)^{*}\right\|_{L^{1}(\partial \Omega)} \leq C\left\|f_{N}\right\|_{H^{1}(N)} .
$$

The constant $C$ above depends on the global character of the domain.

Proof. To begin, let $f_{N}=a$ be an atom for $N$ and let $u$ be the weak solution of the mixed problem with Neumann data $f_{N}$ and zero Dirichlet data. The $H^{1}(N)$ estimate will follow immediately from the estimate for an atom.

We wish to establish a representation formula for the gradient of $u$ in terms of the boundary values of $u$. Let $x \in \Omega$ and let $j$ be an index ranging from 1 to $n$. The claim is that

$$
\begin{array}{r}
\frac{\partial u}{\partial x_{j}}(x)=-\int_{\partial \Omega} \sum_{i=1}^{n} \frac{\partial \Xi}{\partial y_{i}}(x- \\
\cdot)\left(\nu_{i} \frac{\partial u}{\partial y_{j}}-\frac{\partial u}{\partial y_{i}} \nu_{j}\right) \\
+\frac{\partial \Xi}{\partial y_{j}}(x-\cdot) \frac{\partial u}{\partial \nu} d \sigma,
\end{array}
$$

where $\Xi$ is the fundamental solution of the Laplacian. In the case where $u$ is smooth up to the boundary, the formula follows from the divergence theorem. However, it will take more work to prove (4.11) when $u$ is only a weak solution.

Let $\eta$ be a smooth function that is zero in a neighborhood of $\Lambda$ and supported in a coordinate cylinder. Using the coordinates of the coordinate cylinder, let $u_{\tau}(y)=u\left(y+\tau e_{n}\right)$, where $e_{n}$ is the unit vector in the $n$-th direction. Applying the divergence formula gives

$$
\begin{array}{r}
-\int_{\partial \Omega} \eta\left(\sum_{i=1}^{n}\left(\frac{\partial \Xi}{\partial y_{i}}(x-\cdot)\left(\nu_{i} \frac{\partial u_{\tau}}{\partial y_{j}}-\frac{\partial u_{\tau}}{\partial y_{i}} \nu_{j}\right)\right)+\frac{\partial \Xi}{\partial y_{j}}(x-\cdot) \frac{\partial u_{\tau}}{\partial \nu}\right) d \sigma \\
=\eta(x) \frac{\partial u_{\tau}}{\partial x_{j}}(x)-\int_{\Omega} \nabla \eta \cdot \nabla_{y} \Xi(x-\cdot) \frac{\partial u_{\tau}}{\partial y_{j}}-\nabla_{y} \Xi(x-\cdot) \cdot \nabla u_{\tau} \frac{\partial \eta}{\partial y_{j}} \\
+\frac{\partial \Xi}{\partial y_{j}}(x-\cdot) \nabla u_{\tau} \cdot \nabla \eta d y
\end{array}
$$

Since the cutoff function $\eta$ vanishes near $\Lambda$, we may use the truncated maximal function estimate in Lemma 4.4 to let $\tau$ approach 0 from above, and we can conclude that the identity (4.12) continues to hold with $u_{\tau}$ replaced by $u$.

Our next step is to remove the restriction that $\eta$ must vanish on $\Lambda$. Towards this end, suppose that $\eta$ is of the form $\eta=\eta \phi_{t}$, where $\phi_{t}=0$ on the set $\mathcal{C}_{t}, \phi_{t}=1$ on $\Omega \backslash \mathcal{C}_{3 t}$ and $\left|\nabla \phi_{t}(x)\right| \leq C / t$. By our conditions on the dimension of $\Lambda$, (1.3) and Lemma 2.5 we have that if $\lambda<2-\epsilon$, then

$$
\left|\mathcal{C}_{t}\right|<C t^{\lambda}
$$


According to Lemma 3.8, $\nabla u$ lies in $L^{q}(\Omega)$ for $q<q_{0}$. Using Hölder's inequality with $q<q_{0}$, our estimate for $\nabla \phi_{t}$, and (4.13), we obtain

$$
\begin{aligned}
\left|\int_{\Omega} \eta \nabla \phi_{t} \cdot \nabla_{y} \Xi(x-\cdot) \frac{\partial u}{\partial y_{j}} d y\right| & \leq \frac{C\|\eta\|_{L^{\infty}}}{t}\left(\int_{\mathcal{C}_{3 t}}|\nabla u|^{q} d y\right)^{\frac{1}{q}}\left(\int_{\mathcal{C}_{3 t}}|\nabla \Xi|^{q^{\prime}} d y\right)^{\frac{1}{q^{\prime}}} \\
& \leq C t^{-1}\left|\mathcal{C}_{3 t}\right|^{1 / q^{\prime}}\left(\int_{\mathcal{C}_{3 t}}|\nabla u|^{q} d y\right)^{1 / q} \\
& \leq C t^{-1+\lambda / q^{\prime}}\left(\int_{\mathcal{C}_{3 t}}|\nabla u|^{q} d y\right)^{1 / q},
\end{aligned}
$$

where $1 / q+1 / q^{\prime}=1$. If we choose $q$ and $\lambda$ so that $q_{0} /\left(q_{0}-1\right)<q /(q-1)=\lambda<2-\epsilon$, the last term in (4.14) will go to zero as $t$ approaches zero.

The remaining terms in (4.12) can be estimated in a similar manner, yielding

$$
\begin{aligned}
\lim _{t \rightarrow 0^{+}}-\int_{\Omega} \nabla\left(\phi_{t} \eta\right) \cdot \nabla_{y} \Xi(x-\cdot) \frac{\partial u}{\partial y_{j}}-\nabla_{y} \Xi(x-\cdot) \cdot \nabla u \frac{\partial\left(\phi_{t} \eta\right)}{\partial y_{j}} \\
+\frac{\partial \Xi}{\partial y_{j}}(x-\cdot) \nabla u \cdot \nabla\left(\phi_{t} \eta\right) d y \\
=-\int_{\Omega} \nabla \eta \cdot \nabla_{y} \Xi(x-\cdot) \frac{\partial u}{\partial y_{j}}-\nabla_{y} \Xi(x-\cdot) \cdot \nabla u \frac{\partial \eta}{\partial y_{j}} \\
+\frac{\partial \Xi}{\partial y_{j}}(x-\cdot) \nabla u \cdot \nabla \eta d y
\end{aligned}
$$

Thus we obtain (4.12) with $u_{\tau}$ replaced by $u$ and $\eta$ not required to vanish on $\Lambda$. Choose a partition of unity which consists of functions that are either supported in a coordinate cylinder or whose support does not intersect the boundary of $\Omega$. As $\eta$ runs over this partition, the sum gives the representation formula for $\nabla u$ given in (4.11). From Theorem 4.1 we have $\nabla u \in L^{p}(\partial \Omega)$, and by the theorem of Coifman, McIntosh and Meyer [6] it follows that $(\nabla u)^{*} \in L^{p}(\partial \Omega)$ and thus $(\nabla u)^{*} \in L^{1}(\partial \Omega)$ since $\Omega$ is a bounded domain. A few more steps will provide us with the desired estimate, $\left\|(\nabla u)^{*}\right\|_{L^{1}(\partial \Omega)} \leq C$.

Since $(\nabla u)^{*}$ lies in $L^{p}(\partial \Omega)$, we can apply the divergence theorem twice more and obtain the following identities:

$$
\begin{aligned}
\int_{\partial \Omega} \frac{\partial u}{\partial \nu} d \sigma & =0 \\
\int_{\partial \Omega} \nu_{j} \frac{\partial u}{\partial y_{i}}-\nu_{i} \frac{\partial u}{\partial y_{j}} d \sigma & =0 .
\end{aligned}
$$

Using these identities and the estimates for $\nabla u$ established in Theorem 4.1, we can conclude that the two integrands above are molecules on the boundary of the domain, and hence it follows from the representation formula (4.11) that $(\nabla u)^{*}$ lies in $L^{1}(\partial \Omega)$ and satisfies the estimate

$$
\left\|(\nabla u)^{*}\right\|_{L^{1}(\partial \Omega)} \leq C .
$$

The estimate for solutions with Neumann data in $H^{1}(N)$ follows easily from the result for solutions with atomic data. 


\section{UNIQUENESS}

We now turn to establishing uniqueness of solutions to the mixed problem. We rely on the results of the previous section and uniqueness of the regularity problem due to Dahlberg and Kenig [9] (also, see the work of D. Mitrea [23, Corollary 4.2] for the result in two dimensions). More specifically, we prove that if $u$ solves (1.1) with zero data and $(\nabla u)^{*} \in L^{1}(\partial \Omega)$, then $u$ also solves the regularity problem with zero data, and hence $u=0$.

Theorem 5.1. If $u$ is a solution of the $L^{1}$-mixed problem (1.1) with $f_{D}=0$ and $f_{N}=0$, then $u=0$.

The proof of this theorem closely follows the proof of uniqueness in the paper of Ott and Brown [26, Section 5]. We outline the main steps of the argument here and will omit many of the technical details.

Recall the following approximation scheme of G. Verchota ([33, Appendix A] and [34, Theorem 1.12]). Given a Lipschitz domain $\Omega$, there exists a family of smooth domains $\left\{\Omega_{k}\right\}$ with $\bar{\Omega}_{k} \subset \Omega, k=1,2, \ldots$, and a family of bi-Lipschitz homeomorphisms $\Lambda_{k}: \partial \Omega_{k} \rightarrow \partial \Omega$.

The following lemma is proved via the method of Verchota. The argument is sketched in Ott and Brown [26, Section 5]. The proof uses generalized Riesz transforms and also relies on the Hölder continuity of the Green function established in Lemma 3.6. We use a similar argument in Section 7.

Lemma 5.2. Let $\left\{\Omega_{k}\right\}$ be a family of smooth domains. Let $u \in W^{1,1}\left(\partial \Omega_{k}\right)$ for $k=1,2, \ldots$. If $w$ is a weak solution of the mixed problem in $\Omega_{k}$ with Neumann data an atom for $N$ and zero Dirichlet data, then

$$
\left|\int_{\partial \Omega_{k}} u \frac{\partial w}{\partial \nu} d \sigma\right| \leq C_{w}\|u\|_{W^{1,1}\left(\partial \Omega_{k}\right)} .
$$

Next we introduce a Poincaré inequality that will be employed below. Let $N_{\epsilon}=$ $\{x \in N: \delta(x)<\epsilon\}$. We show that there is a constant $C$ such that for $u \in W^{1,1}(\partial \Omega)$, with $u=0$ a.e. on $D$,

$$
\frac{1}{\epsilon} \int_{N_{\epsilon}}|u| d \sigma \leq C \int_{N_{C \epsilon}}|\nabla u| d \sigma
$$

To prove the inequality above, let $x \in N_{\epsilon}$ and let $Q_{x, \epsilon}$ denote the surface cube centered at $x$ with side length $\epsilon$. If $x \in N_{\epsilon}$, then $Q_{x, 2 \epsilon} \cap D \neq \emptyset$. Thus we may use (2.2) to obtain the Poincaré inequality

$$
\int_{Q_{x, 4 \epsilon}}|u| d \sigma \leq C \epsilon \int_{Q_{x, 4 \epsilon}}|\nabla u| d \sigma
$$

By the Besicovitch covering lemma, we can find a finite cover of $N_{\epsilon}$ by cubes $Q_{x_{1}, 4 \epsilon}, Q_{x_{2}, 4 \epsilon}, \ldots, Q_{x_{m}, 4 \epsilon}$ such that the cubes have bounded overlaps,

$$
\sum_{i=1}^{m} \chi_{Q_{x_{i}}, 4 \epsilon} \leq C_{n} .
$$


Then we have

$$
\begin{aligned}
\int_{N_{\epsilon}}|u| d \sigma & \leq \sum_{i=1}^{m} \int_{Q_{x_{i}, 4 \epsilon}}|u| d \sigma \\
& \leq C \sum_{i=1}^{m} \epsilon \int_{Q_{x_{i}, 4 \epsilon}}|\nabla u| d \sigma \\
& \leq C \epsilon \int_{N_{C \epsilon}}|\nabla u| d \sigma .
\end{aligned}
$$

Thus we obtain (5.1), which we will use to prove the following approximation lemma. Let $\Upsilon$ denote the collection of functions defined in $\bar{\Omega}$ that are Lipschitz and compactly supported in $\partial \Omega \backslash \bar{D}$. The next lemma shows that a function $u \in$ $W^{1,1}(\partial \Omega)$ which satisfies $u=0$ a.e. on $D$ can be approximated in the $W^{1,1}(\partial \Omega)$ norm by functions in $\Upsilon$. This density is fairly easy for the domains considered in Ott and Brown [26], but requires more work under the present assumptions.

Lemma 5.3. Let $\Omega$ and $D$ satisfy (1.2) and (2.2). Suppose that $u \in W^{1,1}(\partial \Omega)$ and $u=0$ a.e. on $D$. Then $u$ can be approximated in $W^{1,1}(\partial \Omega)$ by functions from $\Upsilon$.

Proof. Let $u \in W^{1,1}(\partial \Omega)$ and suppose that $u=0$ a.e. on $D$. Fix $\epsilon>0$ and let $\eta_{\epsilon}$ be a smooth function which is 1 if $t>2 \epsilon$ and 0 if $t<\epsilon$, and satisfies $\left|\nabla \eta_{\epsilon}(x)\right|<C / \epsilon$. If $u \in W^{1,1}(\partial \Omega)$ and vanishes a.e. on $D$, then we have that $u_{\epsilon}(x)=\eta_{\epsilon}(\delta(x)) u(x)$ is zero in a neighborhood of $D \cup \Lambda$. Then it follows that $\nabla_{t} u_{\epsilon}-\nabla_{t} u=u \nabla_{t} \eta_{\epsilon}(\delta(\cdot))+\left(\eta_{\epsilon}(\delta(\cdot))-1\right) \nabla_{t} u$. From the dominated convergence theorem,

$$
\lim _{\epsilon \rightarrow 0^{+}} \int_{N}\left|\nabla_{t} u \| \eta_{\epsilon}(\delta(\cdot))-1\right| d \sigma=0 .
$$

Since $\nabla \eta_{\epsilon}(\delta(\cdot)) \leq C / \epsilon$, we may use the Poincaré inequality (5.1), which requires (2.2), and the dominated convergence theorem to conclude that

$$
\lim _{\epsilon \rightarrow 0^{+}} \int_{N}\left|u \nabla_{t} \eta_{\epsilon}(\delta(\cdot))\right| d \sigma=0 .
$$

Thus, we have that $u \in W^{1,1}(\partial \Omega)$ may be approximated by a function $u_{\epsilon}$ that is compactly supported in $\partial \Omega \backslash \bar{D}$. By a standard regularization argument, we can approximate $u_{\epsilon}$ by functions that are in $\Upsilon$.

Proof of Theorem 5.1. Let $u$ be a solution of the mixed problem (1.1) with $f_{N}=0$ and $f_{D}=0$. We wish to show that $u=0$. Fix an atom $a$ for $N$ and let $w$ be a solution of the mixed problem with $f_{N}=a$ and $f_{D}=0$ as constructed in Theorem 4.1. Our goal is to show that

$$
\int_{N} u a d \sigma=0
$$

In turn, this will imply that $u$ is zero on $\partial \Omega$, and by appealing to the uniqueness of the regularity problem proved by Dahlberg and Kenig [9] or D. Mitrea [23] in two dimensions, we can conclude that $u=0$ in $\Omega$. 
To prove (5.2), we apply Green's second identity in one of the smooth approximating domains from Verchota's construction and obtain

$$
\int_{\partial \Omega_{k}} w \frac{\partial u}{\partial \nu} d \sigma=\int_{\partial \Omega_{k}} u \frac{\partial w}{\partial \nu} d \sigma, \quad k=1,2, \ldots
$$

We have that $(\nabla u)^{*} \in L^{1}(\partial \Omega)$, and Lemma 3.6 implies that $w$ is Hölder continuous and hence bounded. Further, $w=0$ on $D$ and $\frac{\partial u}{\partial \nu}=0$ on $N$. Hence by the dominated convergence theorem,

$$
\lim _{k \rightarrow \infty} \int_{\partial \Omega_{k}} w \frac{\partial u}{\partial \nu} d \sigma=0 .
$$

Thus, we can prove our claim by showing that

$$
\lim _{k \rightarrow \infty} \int_{\partial \Omega_{k}} u \frac{\partial w}{\partial \nu} d \sigma=\int_{\partial \Omega} u a d \sigma .
$$

Note that the existence of the limit in (5.5) follows from (5.3) and (5.4). Now by repeating the argument used to prove Lemma 5.7 in the work of Ott and Brown [26], we can find a sequence $\left\{U_{j}\right\}$ of Lipschitz functions defined in $\bar{\Omega}$ such that $\left.U_{j}\right|_{D}=0$ and

$$
\lim _{k \rightarrow \infty}\left\|u-U_{j}\right\|_{W^{1,1}\left(\partial \Omega_{k}\right)} \leq 1 / j .
$$

The argument outlined above uses the density result in Lemma 5.3 . Now we have

$$
\begin{aligned}
\left|\int_{\partial \Omega} u a d \sigma-\lim _{k \rightarrow \infty} \int_{\partial \Omega_{k}} u \frac{\partial w}{\partial \nu} d \sigma\right| \leq \mid & \int_{\partial \Omega} u a d \sigma-\lim _{k \rightarrow \infty} \int_{\partial \Omega_{k}} U_{j} \frac{\partial w}{\partial \nu} d \sigma \mid \\
& +\lim \sup _{k \rightarrow \infty}\left|\int_{\partial \Omega_{k}}\left(u-U_{j}\right) \frac{\partial w}{\partial \nu} d \sigma\right| .
\end{aligned}
$$

Since $(\nabla w)^{*} \in L^{1}(\partial \Omega)$ and $U_{j}$ is bounded, we may take the limit of the first term on the right of (5.7). This yields

$$
\left|\int_{\partial \Omega} u a d \sigma-\lim _{k \rightarrow \infty} \int_{\partial \Omega_{k}} u \frac{\partial w}{\partial \nu} d \sigma\right| \leq\left|\int_{\partial \Omega}\left(u-U_{j}\right) a d \sigma\right|+C / j \leq C / j .
$$

The second term on the right of (5.7) is bounded by $C_{w} / j$ by Lemma $[5.2$ and (5.6). Since $j$ is arbitrary, we have obtained (5.5), and the proof of the theorem is complete.

\section{6. $L^{p}$ RESULT}

In this section, we use the existence of solutions of the mixed problem with data from Hardy spaces established in Section 4 to prove $L^{p}$-estimates for the mixed problem. Our strategy is to first recall the reverse Hölder inequality at the boundary which was proved in Theorem 4.5. With this estimate in hand, we then apply the method developed by Shen 28] and adapted by Ott and Brown 26] to obtain the $L^{p}$-estimate.

The following lemma is a local estimate that is a consequence of Theorem 4.5. In this lemma we use the truncated non-tangential maximal function defined in Section 2 
Lemma 6.1. Let $\Omega$ and $D$ satisfy assumptions (1.2) and (1.4). Let $q_{0}>2$ be as in Lemma 3.8 and let $\Lambda$ satisfy (1.3) with $0 \leq \epsilon<\left(q_{0}-2\right) /\left(q_{0}-1\right)$. Let $u$ be the weak solution of the mixed problem with $f_{N} \in L^{2}(N)$ and zero Dirichlet data. Let $x \in \Omega$ and $0<r<r_{0}$. Then for $1<p<q_{0}((1-\epsilon) /(2-\epsilon))$ the following local estimate holds:

$$
\left(f_{\Delta_{r}(x)}(\nabla u)_{c r}^{* p} d \sigma\right)^{1 / p} \leq C\left(f_{\Psi_{2 r}(x)}|\nabla u| d y+r^{(1-n) / 2}\left\|f_{N}\right\|_{L^{2}\left(\Delta_{2 r}(x) \cap N\right)}\right) .
$$

The constant $c=1 / 16$, and $C$ depends on $M$ and $n$.

Proof. Let $x \in \Omega$ and $r$ satisfy $0<r<r_{0}$. Theorem 4.5 provides an estimate for the $L^{p}$-norm of $\nabla u$ in a surface ball $\Delta_{r}(x)$. To obtain the estimate for the nontangential maximal function, choose a cut-off function $\eta$ which is one on $B_{3 r}(x)$ and supported in $B_{4 r}(x)$. Let $z \in B_{r}(x)$. By repeating the argument used to prove (4.11) in the proof of Theorem 4.7, we can show that the gradient of the weak solution $u$ may be represented as

$$
\begin{gathered}
\left(\eta \frac{\partial u}{\partial z_{j}}\right)(z)=\int_{\partial \Omega} \eta\left(\frac{\partial \Xi}{\partial \nu}(z-\cdot) \frac{\partial u}{\partial y_{j}}-\nu_{j} \nabla_{y} \Xi(z-\cdot) \cdot \nabla u+\frac{\partial \Xi}{\partial y_{j}}(z-\cdot) \frac{\partial u}{\partial \nu}\right) d \sigma \\
-\int_{\Omega} \nabla \eta \cdot \nabla_{y} \Xi(z-\cdot) \frac{\partial u}{\partial y_{j}}-\frac{\partial \eta}{\partial y_{j}} \nabla_{y} \Xi(z-\cdot) \cdot \nabla u \\
+\nabla \eta \cdot \nabla u \frac{\partial \Xi}{\partial y_{j}}(z-\cdot) d y .
\end{gathered}
$$

From this representation and the theorem of Coifman, McIntosh and Meyer [6] on the boundedness of the Cauchy integral, we get

$$
\left(f_{\Delta_{r}(x)}(\nabla u)_{r}^{* p} d \sigma\right)^{1 / p} \leq C\left[f_{\Psi_{4 r}(x)}|\nabla u| d y+\left(f_{\Delta_{4 r}(x)}|\nabla u|^{p} d \sigma\right)^{1 / p}\right]
$$

From estimate (6.1), Theorem 4.5, and a covering argument, we obtain the theorem.

Next, we outline the argument developed by Shen 28 that we employ to obtain $L^{p}$-estimates in this section and weighted $L^{p}$-estimates in the next section. Shen's argument is adapted from work of Peral and Caffarelli [3]. It depends on a CalderónZygmund decomposition of the boundary, and thus we will use surface cubes in this section rather than surface balls $\Delta_{r}(x)$. Before giving Shen's result, recall that a locally integrable function $w$ is an $A_{p}(d \sigma)$ weight, $1<p<\infty$, provided that

$$
\frac{1}{\sigma(\Delta)} \int_{\Delta} w d \sigma\left(\frac{1}{\sigma(\Delta)} \int_{\Delta} w^{-p^{\prime} / p} d \sigma\right)^{p / p^{\prime}} \leq A<\infty
$$

for all surface balls $\Delta \subset \partial \Omega$ centered on $\partial \Omega$. Define $A_{\infty}(d \sigma)=\bigcup_{p} A_{p}(d \sigma)$.

Let $Q_{0}$ be a surface cube and let $F$ be defined on $4 Q_{0}$. Let the exponents $p, q$ satisfy $1<p<q$. Assume that for each $Q \subset Q_{0}$, we may find functions $F_{Q}$ and 
$R_{Q}$, defined in $2 Q$, satisfying

$$
\begin{gathered}
|F| \leq\left|F_{Q}\right|+\left|R_{Q}\right| \\
f_{2 Q}\left|F_{Q}\right| d \sigma \leq C\left(f_{4 Q}|f|^{p} d \sigma\right)^{1 / p}, \\
\left(f_{2 Q}\left|R_{Q}\right|^{q} d \sigma\right)^{1 / q} \leq C\left[f_{4 Q}|F| d \sigma+\left(f_{4 Q}|f|^{p} d \sigma\right)^{1 / p}\right] .
\end{gathered}
$$

Going further, assume that $\mu$ is a weight in $A_{t}(d \sigma)$ and that

$$
\left(\frac{\mu(E)}{\mu(Q)}\right) \leq C\left(\frac{\sigma(E)}{\sigma(Q)}\right)^{\theta}, \quad 1<t<\theta q,
$$

where $\theta$ depends on $M$. Under the assumptions (6.3) $-(6.6)$, for $s$ in the interval $(p, \theta q)$, we have

$$
\left(f_{Q_{0}}|F|^{s} \mu d \sigma\right)^{1 / s} \leq C\left[f_{4 Q_{0}}|F| d \sigma+\left(f_{4 Q_{0}}|f|^{s} \mu d \sigma\right)^{1 / s}\right] .
$$

The constant in the estimate above depends on the Lipschitz constant of $\Omega$ and the constants in the estimates (6.4)-(6.6). The argument to obtain (6.7) is essentially the same as in Shen [28, Theorems 3.2, 3.4]. Ott and Brown [26, Section 7] rework Shen's argument to apply it to the current situation, where our starting point is a result in a Hardy space rather than in an $L^{p}$-space.

Let $4 Q_{0}$ be a surface cube with sidelength comparable to $r_{0}$. Let $u$ be a solution of the mixed problem with Neumann data $f \in L^{p}(N)$ and zero Dirichlet data. Since $L^{p}(N) \subset H^{1}(N)$, we know by Theorem 4.1 that a solution $u$ exists with $(\nabla u)^{*} \in L^{1}(\partial \Omega)$. Let $F=(\nabla u)^{*}$. Now given a cube $Q \subset Q_{0}$ with diameter $r$, we define $F_{Q}$ and $R_{Q}$ as follows. Let $\bar{f}_{4 Q}=0$ if $4 Q \cap D \neq \emptyset$ and $\bar{f}_{4 Q}=f_{4 Q} f d \sigma$ if $4 Q \subset N$. Set $g=\chi_{4 Q}\left(f-\bar{f}_{4 Q}\right)$ and $h=f-g$. By construction, $g$ and $h$ are both elements of $H^{1}(N)$, and thus we may solve the mixed problem with Dirichlet data zero and Neumann data $g$ or $h$. Let $v$ solve the mixed problem with Neumann data equal to $g$ and let $w$ solve the mixed problem with Neumann data $h$. By our uniqueness result Theorem 5.1, we have that $u=v+w$. Let $F_{Q}=(\nabla v)^{*}$ and $R_{Q}=(\nabla w)^{*}$. It follows immediately that (6.3) holds. The procedures for obtaining estimates (6.4) and (6.5) are straightforward and were worked out in detail in Ott and Brown [26].

With (6.3)- 6.5) established, we obtain (6.7) for $s$ in the interval $(p, \theta q)$ and $q<q_{0}((1-\epsilon) /(2-\epsilon))$. From here, we can now easily complete the $L^{p}$-estimate.

Proof of Theorem 1.1. To prove part b), we use Dahlberg and Kenig's result [9, Theorem 4.3] for the regularity problem in Hardy spaces or D. Mitrea's result [23] in two dimensions to reduce to the case where the Dirichlet data is zero. Then we take $f_{N} \in H^{1}(N)$ and use Theorem 4.7 to complete the proof.

To prove part a), consider the mixed problem with zero Dirichlet data and Neumann data in $L^{p}(N)$. Since $L^{p}(N)$ is contained in the Hardy space $H^{1}(N)$, a solution $u$ exists by part b). From the argument of Caffarelli and Peral, as adapted by Shen, and Ott and Brown, we have that $u$ satisfies the estimate

$$
\left\|(\nabla u)^{*}\right\|_{L^{p}(\partial \Omega)} \leq C\left\|f_{N}\right\|_{L^{p}(N)} .
$$

Uniqueness of solutions of the mixed problem follows from Theorem 5.1 


\section{Weighted RESUlT}

In this section we establish results for the mixed problem with data from weighted Sobolev spaces. Throughout this section we assume that $\Omega$ and $D$ satisfy conditions (1.2), (1.3), and (1.4).

To begin, we consider the regularity problem when the data comes from a weighted Sobolev space. We will use the solution of the regularity problem to reduce the study of the mixed problem to the case when the Dirichlet data is zero. The study of the regularity problem contained here is a small extension of work of Shen [27] who studied the regularity problem with data in weighted $L^{2}$-Sobolev spaces. Shen's work is in turn an extension of a method used by Verchota [34 to study the (unweighted) regularity problem in Lipschitz domains. This method is also developed in a recent article of Kilty and Shen 18 that studies the relationship between the regularity problem and the Dirichlet problem for elliptic systems. We choose to repeat well-known arguments for several reasons. Kilty and Shen do not give weighted estimates, and there is a small mistake in [27. The weight defined in equation (7.29) on page 2868 of [27] may not be a doubling weight and hence may not be in any $A_{p}$ class.

The heart of the matter is Lemma 7.3 below, which estimates the normal derivative of a harmonic function in terms of its boundary values. Building toward this result, we begin by recalling that Verchota's result for the regularity problem with data in unweighted Sobolev spaces depends on a duality argument and the solution of the Dirichlet problem with data in weighted $L^{p}$-spaces. Thus, our starting point will be the following result of Dahlberg [7] regarding the weighted Dirichlet problem.

In the results that follow, constants have the dependencies given in Section 2. In addition, the constant may depend on the weight through the $A_{p}$-constant and the exponents appearing in the assumptions on the weights.

We begin by recalling some well-known results about the $L^{p}(\mu d \sigma)$-Dirichlet problem. In this problem, given $f$ on the boundary we look for a function $u$ which satisfies

$$
\begin{cases}\Delta u=0, & \text { in } \Omega, \\ u=f, & \text { on } \partial \Omega, \\ u^{*} \in L^{p}(\mu d \sigma) . & \end{cases}
$$

Theorem 7.1. There exists an exponent $s_{0}<2$ such that if $\mu \in A_{r}(d \sigma), r>1$, $p>r s_{0}$, and $f \in L^{p}(\mu d \sigma)$, then the $L^{p}(\mu d \sigma)$-Dirichlet has a unique solution.

Proof. Dahlberg [7] has shown that there exists an exponent $t_{0}>2$ such that the harmonic measure lies in the reverse Hölder class $\mathcal{B}_{t}(d \sigma)$ for $t<t_{0}$, meaning that for each $t<t_{0}$, there is a constant $C_{t}$ such that

$$
\left(f_{\Delta} \omega^{t} d \sigma\right)^{1 / t} \leq C_{t} f_{\Delta} \omega d \sigma
$$

for any surface ball $\Delta$ centered on $\partial \Omega$. Here, $\omega$ denotes the density with respect to surface measure of harmonic measure at some convenient point in $\Omega$. The exponent $s_{0}$ will be the dual exponent to $t_{0}$, i.e., $1 / s_{0}+1 / t_{0}=1$. 
Let $f \in L^{2}(d \sigma) \cap L^{p}(\mu d \sigma)$, and let $u$ be the solution of the $L^{2}(d \sigma)$-Dirichlet problem. From Hunt and Wheeden [13] (see also Jerison and Kenig [14]), we know that

$$
u^{*}(x) \leq C M_{\omega} f(x),
$$

where $M_{\omega} f$ is the Hardy-Littlewood maximal function with respect to harmonic measure given by

$$
M_{\omega} f(x)=\sup _{r>0} \frac{1}{\omega\left(\Delta_{r}(x)\right)} \int_{\Delta_{r}(x)}|f| \omega d \sigma .
$$

Since $\omega \in \mathcal{B}_{t}(d \sigma)$ for $t<t_{0}$, we have

$$
M_{\omega} f(x) \leq C_{s} M\left(|f|^{s}\right)^{1 / s}(x), \quad s_{0}<s<\infty, 1 / s_{0}+1 / t_{0}=1 .
$$

Next, set $s=p / r$ and note that our assumption $p>r s_{0}$ implies that $s>s_{0}$. Since $\mu \in A_{r}(d \sigma)$, it follows that with this choice of $s$ we have the estimate

$$
\left\|M\left(|f|^{s}\right)^{1 / s}\right\|_{L^{p}(\mu d \sigma)} \leq C\|f\|_{L^{p}(\mu d \sigma)},
$$

which implies that

$$
\left\|u^{*}\right\|_{L^{p}(\mu d \sigma)} \leq C\|f\|_{L^{p}(\mu d \sigma)} .
$$

By a standard limiting argument, we may therefore construct solutions $u \in L^{p}(\mu d \sigma)$ to the Dirichlet problem (7.1) which satisfy the estimate (7.3).

Finally, to establish uniqueness of solutions of (7.1), observe that $L^{p}(\mu d \sigma) \subset$ $L^{s}(d \sigma)$ when $s=p / r$. Since $s>s_{0}$, we may use the uniqueness result for the $L^{s}(d \sigma)$-Dirichlet problem to conclude that if $u$ is harmonic in $\Omega, u^{*} \in L^{p}(\mu d \sigma)$, and $u$ has non-tangential limits of 0 a.e. on $\partial \Omega$, then $u=0$.

The next theorem establishes solvability of the regularity problem when the boundary data lies in a weighted Sobolev space. Given a function $f$ on the boundary, the $L^{p^{\prime}}\left(\mu^{-p^{\prime} / p} d \sigma\right)$-regularity problem is the problem of finding a function $u$ which satisfies

$$
\begin{cases}\Delta u=0, & \text { in } \Omega, \\ u=f, & \text { on } \partial \Omega, \\ (\nabla u)^{*} \in L^{p}\left(\mu^{-p^{\prime} / p} d \sigma\right) . & \end{cases}
$$

Theorem 7.2. Let $s_{0}$ be as in Theorem 7.1 and let $\mu \in A_{r}(d \sigma), r>1$. If $\infty>$ $p>r s_{0}$ and $f$ lies in $W^{1, p^{\prime}}\left(\mu^{-p^{\prime} / p} d \sigma\right)$, then there exists a unique solution of the $L^{p^{\prime}}\left(\mu^{-p^{\prime} / p} d \sigma\right)$-regularity problem with data $f$ which satisfies

$$
\int_{\partial \Omega}\left((\nabla u)^{*}\right)^{p^{\prime}} \mu^{-p^{\prime} / p} d \sigma \leq C \int_{\partial \Omega}\left(\left|\nabla_{t} u\right|^{p^{\prime}}+|u|^{p^{\prime}}\right) \mu^{-p^{\prime} / p} d \sigma .
$$

In the following statement, let $\nabla_{t}$ denote the tangential gradient at the boundary (see Section 2 for the definition).

Lemma 7.3. Let $\mu \in A_{r}(d \sigma)$ with $r>1$ and suppose that $\infty>p>r s_{0}$, where $s_{0}$ is as in Theorem 17.1. If $u$ is a harmonic function with $(\nabla u)^{*} \in L^{2}(d \sigma)$, then

$$
\left\|\frac{\partial u}{\partial \nu}\right\|_{L^{p^{\prime}\left(\mu^{-p^{\prime} / p} d \sigma\right)}} \leq C\left(\left\|\nabla_{t} u\right\|_{L^{p^{\prime}\left(\mu^{-p^{\prime} / p} d \sigma\right)}}+\|u\|_{L^{p^{\prime}\left(\mu^{-p^{\prime} / p} d \sigma\right)}}\right) .
$$


To prove this lemma, we begin by defining local Riesz transforms. Fix a coordinate cylinder $Z_{r}(x), r<r_{0}$, for $\partial \Omega$ such that $Z_{2 r}(x)$ is also a coordinate cylinder. Hereafter in this section we will use $Z_{r}$ to denote $Z_{r}(x)$. Let $\eta$ be a smooth cutoff function such that $\eta=1$ on $Z_{r}$ and $\eta=0$ outside $Z_{3 r / 2}$. Let $v$ be a harmonic function. Using coordinates $\left(x^{\prime}, x_{n}\right) \in \mathbf{R}^{n-1} \times \mathbf{R}$, for $i=1, \ldots, n$, the local Riesz transforms are given by

$$
v_{i}(x)=-\int_{x_{n}}^{\infty} \frac{\partial}{\partial x_{i}}(\eta v)\left(x^{\prime}, t\right) d t
$$

for $x \in Z_{2 r} \cap \Omega$, and $v_{i}(x)=0$ in $\Omega \backslash Z_{2 r}, i=1, \ldots, n$. Straightforward calculations give that

$$
\begin{aligned}
\frac{\partial v_{i}}{\partial x_{j}} & =\frac{\partial v_{j}}{\partial x_{i}} \\
\sum_{i=1}^{n} \frac{\partial v_{i}}{\partial x_{i}}(x) & =-\int_{x_{n}}^{\infty} \nabla \eta\left(x^{\prime}, t\right) \cdot \nabla v\left(x^{\prime}, t\right)+v\left(x^{\prime}, t\right) \Delta \eta\left(x^{\prime}, t\right) d t \\
\Delta v_{i}(x) & =-\int_{x_{n}}^{\infty} \frac{\partial}{\partial x_{i}}\left(\nabla \eta\left(x^{\prime}, t\right) \cdot \nabla v\left(x^{\prime}, t\right)+v\left(x^{\prime}, t\right) \Delta \eta\left(x^{\prime}, t\right)\right) d t
\end{aligned}
$$

Lemma 7.4. Fix $x \in \partial \Omega$ and $0<r<r_{0}$. Let $v_{1}, \ldots, v_{n}$ be the local Riesz transforms of a harmonic function $v$ in a coordinate cylinder $Z_{32 r}$ and suppose that $\mu$ lies in $A_{\infty}(d \sigma)$. Then for $p<\infty$, the following estimate holds:

$$
\int_{\Delta_{r}(x)}\left(v_{i, r}^{*}\right)^{p} \mu d \sigma \leq C\left(\int_{\Delta_{8 r}(x)}\left(v_{8 r}^{*}\right)^{p} \mu d \sigma+\mu\left(\Delta_{8 r}(x)\right) \sup _{K}|v|^{p}\right) .
$$

Above, $K \subset Z_{32 r}$ is a compact subset of $\Omega$ and the cone opening for the nontangential maximal function on the right is larger than the cone opening for the non-tangential maximal function on the left.

Proof. The proof uses a truncated square function which we define by

$$
S_{r}(v)(x)=\left(\int_{\Gamma_{r}(x)}|\nabla v(y)|^{2}|x-y|^{2-n} d y\right)^{1 / 2}
$$

where $\Gamma_{r}(x)$ is a truncated cone as defined in Section 2, Let $v$ be a given harmonic function and then let $v_{i}$ be one of the local Riesz transforms of $v$ defined in a coordinate cylinder $Z_{32 r}$. Write $v_{i}=v_{i}^{\prime}+v_{i}^{\prime \prime}$, where $v_{i}^{\prime}$ is harmonic in $\Omega \cap Z_{16 r}$ and $v_{i}^{\prime \prime}=\Xi *\left(\Delta v_{i} \chi_{Z_{16 r} \cap \Omega}\right)$. We observe that $\Delta v_{i}$ is bounded in $Z_{16 r}$ and

$$
\sup _{\Omega \cap Z_{16 r}}\left|\Delta v_{i}\right| \leq \frac{C}{r^{2}} \sup _{K}|v|,
$$

where $K$ is the compact set

$$
K=\left\{\left(y^{\prime}, y_{n}\right):\left|y^{\prime}-x^{\prime}\right| \leq 32 r,(1+M) r \leq y_{n}-x_{n} \leq(1+M) 32 r\right\}
$$


Thus, we have

$$
\sup _{Z_{16 r}}\left|v_{i}^{\prime \prime}\right|+r\left|\nabla v_{i}^{\prime \prime}\right| \leq C \sup _{K}|v|
$$

With these preliminaries, we can now give the main estimate,

$$
\begin{aligned}
\int_{\Delta_{r}(x)}\left(v_{i, r}^{*}\right)^{p} \mu d \sigma & \leq C\left(\int_{\Delta_{r}(x)}\left(v_{i, r}^{\prime}{ }^{*}\right)^{p} \mu d \sigma+\mu\left(\Delta_{r}(x)\right) \sup _{K}|v|^{p}\right) \\
& \leq C\left(\int_{\Delta_{2 r}(x)} S_{2 r}\left(v_{i}^{\prime}\right)^{p} \mu d \sigma+\mu\left(\Delta_{r}(x)\right) \sup _{K}|v|^{p}\right) \\
& \leq C\left(\int_{\Delta_{4 r}(x)} S_{4 r}\left(v_{n}^{\prime}\right)^{p} \mu d \sigma+\mu\left(\Delta_{r}(x)\right) \sup _{K}|v|^{p}\right) \\
& \leq C\left(\int_{\Delta_{8 r}(x)}\left(v_{n, 8 r^{*}}\right)^{p} \mu d \sigma+\mu\left(\Delta_{r}(x)\right) \sup _{K}|v|^{p}\right) .
\end{aligned}
$$

The first inequality follows from (7.6), the second is a local version of a theorem of Dahlberg [8, Theorem 1], the third inequality follows from a pointwise estimate which may be found in Stein [30, pp. 213-214], and finally the fourth inequality follows from Dahlberg's result and (7.6). Note that in each of the inequalities above, the cone opening for the object on the left side must be smaller than the cone opening for the object on the right side. Our notation is already elaborate, and thus, we choose to suppress this dependence. Once we recall that $v_{n}=\eta v$, then the estimate of the lemma follows.

We now are ready to present a proof of Lemma 7.3 .

Proof of Lemma [7.3. Let $u$ be a solution of the $L^{2}(d \sigma)$-regularity problem with data $f \in W^{1,2}(d \sigma)$. Since we may solve the $L^{2}(d \sigma)$-regularity problem [14, we may assume that $f$ is supported in a surface ball $\Delta_{r}(x), x \in \partial \Omega$, and that $Z_{32 r}=Z_{32 r}(x)$ is a coordinate cylinder. Suppose that $\partial \Omega$ is given as the graph of $\phi$ in $Z_{32 r}$. We would like to show that

$$
\left\|\frac{\partial u}{\partial \nu}\right\|_{L^{p^{\prime}\left(\mu^{-p^{\prime} / p} d \sigma\right)}} \leq C\|u\|_{W^{1, p^{\prime}\left(\mu^{-p^{\prime} / p} d \sigma\right)}} .
$$

Toward this end, choose $g \in W^{1,2}(d \sigma)$ and let $v$ be the solution of the Dirichlet problem with data $g$. We observe that

$$
\left\|\frac{\partial u}{\partial \nu}\right\|_{L^{p^{\prime}\left(\mu^{-p^{\prime} / p} d \sigma\right)}}=\sup _{\|g\|_{L^{p}(\mu d \sigma)} \leq 1} \int_{\partial \Omega} g \frac{\partial u}{\partial \nu} d \sigma .
$$

Since $v=g$ on $\partial \Omega$, $u$ and $v$ are harmonic, and $(\nabla u)^{*}+(\nabla v)^{*} \in L^{2}(d \sigma)$, we may use Green's second identity to conclude that

$$
\int_{\partial \Omega} g \frac{\partial u}{\partial \nu} d \sigma=\int_{\partial \Omega} u \frac{\partial v}{\partial \nu} d \sigma
$$


As $u=0$ on $\partial \Omega \backslash Z_{r}$, we may use that $v_{n}=\eta v=v$ on $Z_{r}$, (7.4), (7.5), some algebra, and integration by parts to obtain

$$
\begin{aligned}
\int_{\partial \Omega} u \frac{\partial v}{\partial \nu} d \sigma & =\int_{\partial \Omega} u \frac{\partial v_{n}}{\partial \nu} d \sigma \\
& =\int_{\mathbf{R}^{n-1}} u\left(x^{\prime}, \phi\left(x^{\prime}\right)\right)\left(-\frac{\partial v_{n}}{\partial x_{n}}\left(x^{\prime}, \phi\left(x^{\prime}\right)\right)\right. \\
& \left.\quad+\sum_{i=1}^{n-1} \phi_{x_{i}} \frac{\partial v_{n}}{\partial x_{i}}\left(x^{\prime}, \phi\left(x^{\prime}\right)\right)\right) d x^{\prime} \\
= & \int_{\mathbf{R}^{n-1}} u\left(x^{\prime}, \phi\left(x^{\prime}\right)\right)\left(F\left(x^{\prime}, \phi\left(x^{\prime}\right)\right)+\sum_{i=1}^{n-1} \frac{\partial}{\partial x_{i}} v_{i}\left(x^{\prime}, \phi\left(x^{\prime}\right)\right)\right) d x^{\prime} \\
= & \int_{\partial \Omega}\left(u F-\sum_{i=1}^{n-1} v_{i} \frac{\partial u}{\partial \tau_{i}}\right) d \sigma,
\end{aligned}
$$

where $\tau_{i}=\left(1+|\nabla \phi|^{2}\right)^{-1 / 2}\left(e_{i}+\phi_{x_{i}} e_{n}\right)$ is a tangential vector, $v_{i}$ are the local Riesz transforms, and $F=\sum_{i=1}^{n} \frac{\partial v_{i}}{\partial x_{i}}$ is the right-hand side of (17.5). Thus from Lemma 7.4 and Theorem 7.1, it follows that

$$
\begin{aligned}
\left|\int_{\partial \Omega} u \frac{\partial v}{\partial \nu} d \sigma\right| & \leq\|u\|_{L^{1}(d \sigma)}\|F\|_{L^{\infty}(d \sigma)}+\left\|v^{*}\right\|_{L^{p}(\mu d \sigma)}\left\|\nabla_{t} u\right\|_{L^{p^{\prime}\left(\mu^{-p^{\prime} / p} d \sigma\right)}} \\
& \leq C\|u\|_{W^{1, p^{\prime}\left(\mu^{-p^{\prime} / p} d \sigma\right)}}\|g\|_{L^{p}(\mu d \sigma)} .
\end{aligned}
$$

We give the details for the estimate for the term $\|u\|_{L^{1}(d \sigma)}\|F\|_{L^{\infty}(d \sigma)}$. By a Poincaré inequality and Hölder's inequality, we obtain

$$
\|u\|_{L^{1}(d \sigma)} \leq C r\left\|\nabla_{t} u\right\|_{L^{1}(d \sigma)} \leq C r\left\|\nabla_{t} u\right\|_{L^{p^{\prime}\left(\mu^{-p^{\prime} / p} d \sigma\right)}} \mu\left(\Delta_{r}(x)\right)^{1 / p} .
$$

Recall that $F$ is the right-hand side of (7.5). Then it follows that

$$
\|F\|_{L^{\infty}(d \sigma)} \leq C r^{-1} \sup _{K}|v| \leq C r^{-1} \mu\left(\Delta_{r}(x)\right)^{-1 / p}\left\|v^{*}\right\|_{L^{p}(\mu d \sigma)} .
$$

With these inequalities, the stated estimate follows.

Before proving Theorem 7.2 we require one more standard lemma.

Lemma 7.5. If $\mu \in A_{r}(d \sigma), 1<r<\infty, \Delta u=0$, and $(\nabla u)^{*} \in L^{2}(d \sigma)$, then

$$
\int_{\partial \Omega}\left((\nabla u)^{*}\right)^{r} \mu d \sigma \leq C \int_{\partial \Omega}|\nabla u|^{r} \mu d \sigma .
$$

Proof. Let $\Xi$ be the fundamental solution for the Laplacian. We assume that $(\nabla u)^{*} \in L^{2}(d \sigma)$. Under these conditions, it is easy to establish the representation formula

$$
\frac{\partial u}{\partial x_{j}}(x)=-\int_{\partial \Omega} \frac{\partial \Xi}{\partial y_{i}}(x-\cdot)\left(\nu_{i} \frac{\partial u}{\partial y_{j}}-\nu_{j} \frac{\partial u}{\partial y_{i}}\right)+\frac{\partial \Xi}{\partial y_{i}}(x-\cdot) \frac{\partial u}{\partial \nu} d \sigma .
$$

The lemma now follows from standard estimates on singular integral operators on Lipschitz surfaces [6] and weighted estimates for Calderón-Zygmund operators [5].

We are now prepared to prove Theorem 7.2 
Proof of Theorem 7.2. Let $f \in W^{1, p^{\prime}}\left(\mu^{-p^{\prime} / p} d \sigma\right) \cap W^{1,2}(d \sigma)$ and let $u$ be the solution of the $L^{2}(d \sigma)$-regularity problem with data $f$. From Lemma 7.3 and Lemma 7.5. we conclude that

$$
\int_{\partial \Omega}\left((\nabla u)^{*}\right)^{p^{\prime}} \mu^{-p^{\prime} / p} d \sigma \leq C \int_{\partial \Omega}\left(\left|\nabla_{t} u\right|^{p^{\prime}}+|u|^{p^{\prime}}\right) \mu^{-p^{\prime} / p} d \sigma
$$

Now a limiting argument gives existence of solutions of the regularity problem with boundary data $f \in W^{1, p^{\prime}}\left(\mu^{-p^{\prime} / p} d \sigma\right)$. Since $L^{p^{\prime}}\left(\mu^{-p^{\prime} / p} d \sigma\right) \subset L^{1}(d \sigma)$, uniqueness follows from Dahlberg and Kenig 9 .

Theorem 7.6. Let $t_{0}$ be as in the reverse Hölder inequality (7.2) and $s_{0}=t_{0} /\left(t_{0}-1\right)$. Suppose that $\mu \in A_{r}(d \sigma)$, where $p>r s_{0}$. Set

$$
\alpha=\frac{p-1}{r-1} \quad \text { and } \quad \theta=\frac{1}{\alpha^{\prime}}=1-\frac{r-1}{p-1}=\frac{p-r}{p-1} .
$$

Assume that $1<p^{\prime}<\theta\left(q_{0} / 2\right)$. Consider the mixed problem with Dirichlet data $f_{D} \in W^{1, p^{\prime}}\left(\mu^{-p^{\prime} / p} d \sigma\right)$ and Neumann data $f_{N} \in L^{p^{\prime}}\left(\mu^{-p^{\prime} / p} d \sigma\right)$. Then the following estimate for the solution $u$ of the mixed problem holds:

$$
\left\|(\nabla u)^{*}\right\|_{L^{p^{\prime}\left(\mu^{-p^{\prime} / p} d \sigma\right)}} \leq C\left(\left\|f_{N}\right\|_{L^{p^{\prime}}\left(\mu^{-p^{\prime} / p} d \sigma\right)}+\left\|f_{D}\right\|_{W^{1, p^{\prime}}\left(\mu^{-p^{\prime} / p} d \sigma\right)}\right) .
$$

Furthermore, there is only one solution satisfying $(\nabla u)^{*} \in L^{p^{\prime}}\left(\mu^{-p^{\prime} / p} d \sigma\right)$.

Proof. In our application, we are given $\mu \in A_{r}(d \sigma)$ and $p>r s_{0}$. We will apply (6.7) with $\mu^{-p^{\prime} / p} \in A_{p^{\prime}}(d \sigma)$, and we observe that with $\alpha=\frac{p-1}{r-1}$ we have

$$
\begin{aligned}
\int_{\Delta}\left(\mu^{-p^{\prime} / p}\right)^{\alpha} d \sigma & =\int_{\Delta} \mu^{-1 /(r-1)} d \sigma \\
& \leq\left(\int_{\Delta} \mu d \sigma\right)^{-1 /(r-1)} \sigma(\Delta)^{r^{\prime}} \\
& \leq\left(\int_{\Delta} \mu^{-p^{\prime} / p} d \sigma\right)^{(p-1) /(r-1)} \sigma(\Delta)^{r^{\prime}} \sigma(\Delta)^{-p^{\prime} /(r-1)},
\end{aligned}
$$

for any surface ball $\Delta$. Therefore,

$$
f_{\Delta} \mu^{\left(-p^{\prime} \alpha\right) / p} d \sigma \leq\left(f_{\Delta} \mu^{-p^{\prime} / p} d \sigma\right)^{\alpha}
$$

where we have used the $A_{r}$ condition for $\mu$,

$$
\left(\int_{\Delta} \mu d \sigma\right)^{1 /(r-1)}\left(\int_{\Delta} \mu^{-1 /(r-1)} d \sigma\right) \leq \sigma(\Delta)^{r^{\prime}}
$$

and Hölder's inequality,

$$
\sigma(\Delta)^{1 /(r-1)} \leq\left(\int_{\Delta} \mu d \sigma\right)^{p /(r-1)}\left(\int_{\Delta} \mu^{-p^{\prime} / p} d \sigma\right)^{(p-1) /(r-1)} .
$$

From the inequality (7.8) we have $\mu^{-p^{\prime} / p} \in \mathcal{B}_{\alpha}(d \sigma)$, and Hölder's inequality implies

$$
\left(\frac{\mu(E)}{\mu(\Delta)}\right) \leq\left(\frac{\sigma(E)}{\sigma(\Delta)}\right)^{\theta}, \quad \theta=1-\frac{1}{\alpha}=\frac{p-r}{p-1} .
$$

Thus, we have the conditions needed to obtain the conclusion (6.7). 
To summarize, if $1<p^{\prime}<\theta q_{0} / 2$, with $q_{0}$ as in Lemma 3.8, and if $\mu \in A_{r}(d \sigma)$, $p>r s_{0}$, with $s_{0}$ as in Theorem 7.1, then we have the solution of the mixed problem satisfies

$$
\left\|(\nabla u)^{*}\right\|_{L^{p}\left(\mu^{-p^{\prime} / p} d \sigma\right)} \leq C\left(\left\|f_{N}\right\|_{L^{p^{\prime}\left(\mu^{-p^{\prime} / p} d \sigma\right)}}+\left\|f_{D}\right\|_{\left.W^{1, p^{\prime}\left(\mu^{-p^{\prime} / p} d \sigma\right)}\right)}\right) .
$$

Since $L^{p^{\prime}}\left(\mu^{-p^{\prime} / p}\right) \subset L^{1}(d \sigma)$, uniqueness for solutions of the mixed problem with $(\nabla u)^{*} \in L^{p^{\prime}}\left(\mu^{-p^{\prime} / p} d \sigma\right)$ follows from part a) of Theorem 1.1.

\section{ACKNOWLEDGMENT}

The authors thank the referee for helpful remarks. Part of this work was carried out while the third author was visiting the Mathematical Sciences Research Institute in Berkeley, California, whose hospitality is gratefully acknowledged.

\section{REFERENCES}

[1] C. Băcuţă, A.L. Mazzucato, V. Nistor, and L. Zikatanov. Interface and mixed boundary value problems on $n$-dimensional polyhedral domains. Doc. Math., 15:687-745, 2010. MR2735986

[2] R.M. Brown. The mixed problem for Laplace's equation in a class of Lipschitz domains. Comm. Partial Diff. Eqns., 19:1217-1233, 1994. MR1284808 (95i:35059)

[3] L.A. Caffarelli and I. Peral. On $W^{1, p}$ estimates for elliptic equations in divergence form. Comm. Pure Appl. Math., 51(1):1-21, 1998. MR1486629 (99c:35053)

[4] S. Chanillo and Y.Y. Li. Continuity of solutions of uniformly elliptic equations in $\mathbf{R}^{2}$. Manuscripta Math., 77(4):415-433, 1992. MR1190215 (93k:35033)

[5] R.R. Coifman and C. Fefferman. Weighted norm inequalities for maximal functions and singular integrals. Studia Math., 51:241-250, 1974. MR0358205 (50:10670)

[6] R.R. Coifman, A. McIntosh, and Y. Meyer. L'intégrale de Cauchy définit un opérateur borné sur $L^{2}$ pour les courbes lipschitziennes. Ann. of Math., 116:361-387, 1982. MR672839 (84m:42027)

[7] B.E.J. Dahlberg. Estimates of harmonic measure. Arch. Rational Mech. Anal., 65(3):275-288, 1977. MR0466593 (57:6470)

[8] B.E.J. Dahlberg. Weighted norm inequalities for the Lusin area integral and nontangential maximal functions for functions harmonic in a Lipschitz domain. Studia Math., 67:297-314, 1980. MR592391 (82f:31003)

[9] B.E.J. Dahlberg and C.E. Kenig. Hardy spaces and the Neumann problem in $L^{p}$ for Laplace's equation in Lipschitz domains. Ann. of Math., 125:437-466, 1987. MR890159 (88d:35044)

[10] E. De Giorgi. Sulla differenziabilità e l'analiticità delle estremali degli integrali multipli regolari. Mem. Accad. Sci. Torino. Cl. Sci. Fis. Mat. Nat. (3), 3:25-43, 1957. MR0093649 $(20: 172)$

[11] K. Gröger. A $W^{1, p}$-estimate for solutions to mixed boundary value problems for second order elliptic differential equations. Math. Ann., 283(4):679-687, 1989. MR.990595 (90d:35035)

[12] M. Grüter and K.O. Widman. The Green function for uniformly elliptic equations. Manuscripta Math., 37(3):303-342, 1982. MR657523(83h:35033)

[13] R.A. Hunt and R.L. Wheeden. Positive harmonic functions on Lipschitz domains. Trans. Amer. Math. Soc., 147:507-527, 1970. MR0274787 (43:547)

[14] D.S. Jerison and C.E. Kenig. Boundary value problems in Lipschitz domains. In Walter Littman, editor, Studies in partial differential equations, volume 23 of MAA Studies in Mathematics, pages 1-68. Math. Assoc. Amer., Washington, D.C., 1982. MR716504 (85f:35057)

[15] D.S. Jerison and C.E. Kenig. The Neumann problem on Lipschitz domains. Bull. Amer. Math. Soc., 4:203-207, 1982. MR598688 (84a:35064)

[16] C.E. Kenig. Harmonic analysis techniques for second order elliptic boundary value problems. Published for the Conference Board of the Mathematical Sciences, Washington, DC, 1994. MR.1282720(96a:35040)

[17] C.E. Kenig and W.M. Ni. On the elliptic equation $L u-k+K \exp [2 u]=0$. Ann. Scuola Norm. Sup. Pisa Cl. Sci. (4), 12(2):191-224, 1985. MR829052 (87f:35065)

[18] J. Kilty and Z. Shen. The $L^{p}$ regularity problem on Lipschitz domains. Trans. Amer. Math. Soc., 363(3):1241-1264, 2011. MR2737264 (2012a:35072) 
[19] O.A. Ladyzhenskaya and N.N. Ural'tseva. Linear and quasilinear elliptic equations. Translated from the Russian by Scripta Technica, Inc. Translation editor: Leon Ehrenpreis. Academic Press, New York, 1968. MR0244627 (39:5941)

[20] L. Lanzani, L. Capogna, and R.M. Brown. The mixed problem in $L^{p}$ for some two-dimensional Lipschitz domains. Math. Ann., 342(1):91-124, 2008. MR2415316 (2009c:35051)

[21] J. Lehrbäck. Weighted Hardy inequalities and the size of the boundary. Manuscripta Math., 127(2):249-273, 2008. MR2442898 (2009g:46063)

[22] N.G. Meyers. An $L^{p}$-estimate for the gradient of solutions of second order elliptic divergence equations. Ann. Scuola Norm. Sup. Pisa (3), 17:189-206, 1963. MR0159110 (28:2328)

[23] D. Mitrea. Layer potentials and Hodge decompositions in two dimensional Lipschitz domains. Math. Ann., 322(1):75-101, 2002. MR1883390 (2003d:42024)

[24] I. Mitrea and M. Mitrea. The Poisson problem with mixed boundary conditions in Sobolev and Besov spaces in non-smooth domains. Trans. Amer. Math. Soc., 359(9):4143-4182 (electronic), 2007. MR2309180 (2008e:35031)

[25] J. Moser. On Harnack's theorem for elliptic differential operators. Comm. Pure Appl. Math., 14:577-591, 1961. MR0159138 (28:2356)

[26] K.A. Ott and R.M. Brown. The mixed problem for the Laplacian in Lipschitz domains. arXiv:0909.0061 [math.AP], 2009. To appear in Potential Analysis, DOI 10.1007/s11118-0129317-6.

[27] Z. Shen. Weighted estimates in $L^{2}$ for Laplace's equation on Lipschitz domains. Trans. Amer. Math. Soc., 357:2843-2870, 2005. MR2139930(2006b:35074)

[28] Z. Shen. The $L^{p}$ boundary value problems on Lipschitz domains. Adv. Math., 216(1):212-254, 2007. MR2353255 (2009a:35064)

[29] G. Stampacchia. Problemi al contorno ellitici, con dati discontinui, dotati di soluzionie hölderiane. Ann. Mat. Pura Appl. (4), 51:1-37, 1960. MR0126601 (23:A3897)

[30] E.M. Stein. Singular integrals and differentiability properties of functions. Princeton University Press, Princeton NJ, 1970. MR.0290095 (44:7280)

[31] J.D. Sykes and R.M. Brown. The mixed boundary problem in $L^{p}$ and Hardy spaces for Laplace's equation on a Lipschitz domain. In Harmonic analysis and boundary value problems (Fayetteville, AR, 2000), volume 277 of Contemp. Math., pages 1-18. Amer. Math. Soc., Providence, RI, 2001. MR,1840423 (2002g:35058)

[32] M. Venouziu and G.C. Verchota. The mixed problem for harmonic functions in polyhedra of $\mathbf{R}^{3}$. Perspectives in partial differential equations, harmonic analysis and applications, Proc. Sympos. Pure Math., volume 79, pages 407-423. Amer. Math. Soc., Providence, RI, 2008. MR2500502 (2010g:35054)

[33] G.C. Verchota. Layer potentials and boundary value problems for Laplace's equation on Lipschitz domains. Ph.D. thesis, University of Minnesota, 1982. MR2632050

[34] G.C. Verchota. Layer potentials and regularity for the Dirichlet problem for Laplace's equation on Lipschitz domains. J. Funct. Anal., 59:572-611, 1984. MR769382 (86e:35038) 3341

Department of Mathematics, Murray State University, Murray, Kentucky 42071-

E-mail address: jtaylor52@murraystate.edu

Department of Mathematics, University of Kentucky, Lexington, Kentucky 405060027

E-mail address: katharine.ott@uky.edu 0027

Department of Mathematics, University of Kentucky, Lexington, Kentucky 40506-

E-mail address: russell.brown@uky.edu 